\title{
Article
}

\section{Electronic correlations in FeGa3 and the effect of hole doping on its magnetic properties}

Gamza, M. B., Tomczak, J. M., Brown, C., Puri, A., Kotliar, G. and Aronson, M. C.

Available at https://clok.uclan.ac.uk/11705/

Gamza, M. B. orcid iconORCID: 0000-0003-3360-4006, Tomczak, J. M., Brown, C., Puri, A., Kotliar, G. and Aronson, M. C. (2014) Electronic correlations in FeGa3 and the effect of hole doping on its magnetic properties. Physical Review B, 89 (19). p. 195102. ISSN 1098-0121

It is advisable to refer to the publisher's version if you intend to cite from the work. http://dx.doi.org/10.1103/PhysRevB.89.195102

For more information about UCLan's research in this area go to http://www.uclan.ac.uk/researchgroups/ and search for <name of research Group>.

For information about Research generally at UCLan please go to http://www.uclan.ac.uk/research/

All outputs in CLoK are protected by Intellectual Property Rights law, including Copyright law. Copyright, IPR and Moral Rights for the works on this site are retained by the individual authors and/or other copyright owners. Terms and conditions for use of this material are defined in the policies page.

\section{CLoK}

Central Lancashire online Knowledge www.clok.uclan.ac.uk

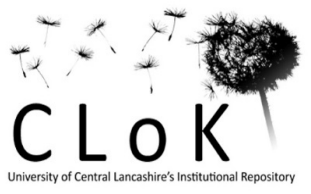




\title{
Electronic correlations in $\mathrm{FeGa}_{3}$ and the effect of hole doping on its magnetic properties
}

\author{
M. B. Gamża, ${ }^{1, *}$ J. M. Tomczak, ${ }^{2, \dagger}$ C. Brown, ${ }^{3,4}$ A. Puri,,${ }^{1,5}$ G. Kotliar, ${ }^{2}$ and M. C. Aronson ${ }^{1,5}$ \\ ${ }^{1}$ Condensed Matter Physics and Materials Science Department, Brookhaven National Laboratory, Upton, New York 11973-5000, USA \\ ${ }^{2}$ Department of Physics and Astronomy, Rutgers University, Piscataway, New Jersey 08854, USA \\ ${ }^{3}$ NIST Center for Neutron Research, National Institute of Standards and Technology, Gaithersburg, Maryland 20899, USA \\ ${ }^{4}$ Department of Chemical and Biomolecular Engineering, University of Delaware, Delaware 19716, USA \\ ${ }^{5}$ Department of Physics and Astronomy, State University of New York, Stony Brook, New York 11794-3800, USA
}

(Received 13 January 2014; revised manuscript received 14 April 2014; published 6 May 2014)

\begin{abstract}
We investigate signatures of electronic correlations in the narrow-gap semiconductor $\mathrm{FeGa}_{3}$ by means of electrical resistivity and thermodynamic measurements performed on single crystals of $\mathrm{FeGa}_{3}, \mathrm{Fe}_{1-x} \mathrm{Mn}_{x} \mathrm{Ga}_{3}$, and $\mathrm{FeGa}_{3-y} \mathrm{Zn}_{y}$, complemented by a study of the $4 d$ analog material $\mathrm{RuGa}_{3}$. We find that the inclusion of sizable amounts of $\mathrm{Mn}$ and $\mathrm{Zn}$ dopants into $\mathrm{FeGa}_{3}$ does not induce an insulator-to-metal transition. Our study indicates that both substitution of $\mathrm{Zn}$ onto the $\mathrm{Ga}$ site and replacement of Fe by $\mathrm{Mn}$ introduces states into the semiconducting gap that remain localized even at highest doping levels. Most importantly, using neutron powder diffraction measurements, we establish that $\mathrm{FeGa}_{3}$ orders magnetically above room temperature in a complex structure, which is almost unaffected by the doping with $\mathrm{Mn}$ and $\mathrm{Zn}$. Using realistic many-body calculations within the framework of dynamical mean field theory (DMFT), we argue that while the iron atoms in $\mathrm{FeGa}_{3}$ are dominantly in an $S=1$ state, there are strong charge and spin fluctuations on short-time scales, which are independent of temperature. Further, the low magnitude of local contributions to the spin susceptibility advocates an itinerant mechanism for the spin response in $\mathrm{FeGa}_{3}$. Our joint experimental and theoretical investigations classify $\mathrm{FeGa}_{3}$ as a correlated band insulator with only small dynamical correlation effects, in which nonlocal exchange interactions are responsible for the spin gap of $0.4 \mathrm{eV}$ and the antiferromagnetic order. We show that hole doping of $\mathrm{FeGa}_{3}$ leads, within DMFT, to a notable strengthening of many-body renormalizations.
\end{abstract}

DOI: 10.1103/PhysRevB.89.195102

PACS number(s): 75.50.Pp, 72.20.-i, 72.80.Ga, 71.20.-b

\section{INTRODUCTION}

The role of electron-electron correlation effects in narrow-gap $d$-electron semiconductors has been a subject of extensive study for over two decades [1-37]. For archetypal compounds of this family, $\mathrm{FeSi}$ and $\mathrm{FeSb}_{2}$, an intriguing crossover was observed from a nonmagnetic semiconductor at low temperatures to a paramagnetic metal with a CurieWeiss-type magnetic susceptibility at high temperatures that are, however, still small relative to the gap energy [1-3]. Furthermore, when passing this crossover, optical spectral weight that is suppressed upon cooling due to the opening of the gap is recovered only at energies that are very high with respect to the charge gap. These distinct similarities of $\mathrm{FeSi}$ and $\mathrm{FeSb}_{2}$ to heavy-fermion Kondo insulators [38] in both charge and spin degrees of freedom have caused great interest in this class of materials.

Models that were proposed to explain the unusual behaviors of $\mathrm{FeSi}$ and $\mathrm{FeSb}_{2}$ include a nearly magnetic semiconductor scenario based on a spin fluctuation theory of itinerant electrons [4-6] and, alternatively, a renormalization of the electronic structure mediated by a strong electronphonon coupling [7]. Recent studies tend to treat $\mathrm{FeSi}$ and $\mathrm{FeSb}_{2}$ as correlated band insulators [8-15]. Unlike conventional semiconductors, the metallization of correlated band

*Current address: Department of Physics, Royal Holloway, University of London, Egham, TW20 0EX, UK.

${ }^{\dagger}$ Current address: Institute of Solid State Physics, Vienna University of Technology, 1040 Wien, Austria. insulators is believed to be caused by the emergence of incoherent states in the gap, accompanied by a massive reorganization of the spin excitation spectrum [10]. For FeSi, this description led to a coherence-incoherence scenario in which the temperature-induced metallization is associated with the unlocking of fluctuating iron moments that are almost temperature independent on short-time scales [10]. It remains to be seen whether the latter paradigm is applicable to other compounds, motivating these investigations of $\mathrm{FeGa}_{3}$.

Early reports on $\mathrm{FeGa}_{3}$ classify the compound as a diamagnetic semiconductor with a narrow gap of 0.4$0.5 \mathrm{eV}[31,32,39-42]$. The magnetic susceptibility increases strongly at temperatures above $\sim 500 \mathrm{~K}$ [39], suggestive of an approaching crossover to a paramagnetic metallic state. Electronic band-structure calculations within the local density approximation (LDA) find a gap of $0.4 \mathrm{eV}$ in the ground state that is produced by the hybridization of $\mathrm{Fe} 3 d$ states with $p$ states of Ga $[31,33,36,41]$, in good agreement with experimental results [31,32]. However, an angle-resolved photoemission spectroscopy (ARPES) study disclosed differences between the measured electronic dispersions and those obtained in the LDA calculations [31], suggesting a band narrowing due to electronic correlations. With these findings, $\mathrm{FeGa}_{3}$ is an appealing compound for studying the consequences of electron-electron correlation effects in a $d$-electron semiconductor, in which the band gap is about one order of magnitude larger than in $\mathrm{FeSi}$ [17] and $\mathrm{FeSb}_{2}[2,3]$.

Similar to those latter compounds, the presence of narrow iron $3 d$ states near the Fermi level raises the question of whether there are local $\mathrm{Fe}$ moments and/or magnetic order in 
$\mathrm{FeGa}_{3}$. So far, there is no consensus in either experimental or theoretical studies as to the overall magnetic character of $\mathrm{FeGa}_{3}[31,33,36,43-45]$. LSDA $+U$ electronic-structure calculations find the existence of local Fe moments in $\mathrm{FeGa}_{3}$, independent of which double-counting scheme is used, with an antiferromagnetic order being lowest in energy [33]. Further, the size of the band gap obtained assuming moderate values of the onsite Coulomb interaction for the Fe $3 d$ states $U \sim$ $2 \mathrm{eV}$ coincides with experimental results [33]. However, a nonmagnetic state is stabilized for $U \lesssim 1.5 \mathrm{eV}$ and even for larger values of $U$ if screening effects are included in the LSDA $+U$ formalism via a Yukawa ansatz [36]. On the experimental side, a recent muon spin rotation study detected spectroscopic features characteristic of electron confinement into spin polarons [44]. Formation of spin polarons requires the existence of Fe moments, whereas a ${ }^{57} \mathrm{Fe}$ Mössbauer study did not show the presence of an internal magnetic field at the $\mathrm{Fe}$ site, indicating a nonmagnetic state of $\mathrm{Fe}$ [45].

In this work, we address the magnetic properties of $\mathrm{FeGa}_{3}$ using neutron powder diffraction experiments. The diffraction patterns reveal the presence of ordered Fe moments, featuring a complex room-temperature magnetic structure. We performed thermodynamic and electrical resistivity measurements on single crystals of $\mathrm{FeGa}_{3}$ and of its isostructural homolog $\mathrm{RuGa}_{3}$. A comparative study of the magnetic properties and the electrical resistivities of these compounds allows us to separate the contributions originating from strongly correlated $3 d$ electrons. Analysis of the specific heat of $\mathrm{FeGa}_{3}$ gives us insight into the vibrational properties of $\mathrm{Fe}$ and $\mathrm{Ga}$ sublattices. The experimental investigation is complemented by electronicstructure calculations based on dynamical mean field theory (DMFT) aimed at exploring the charge and spin states of iron in $\mathrm{FeGa}_{3}$ and effects of many-body renormalizations.

A characteristic feature of correlated insulators is a strong suppression of the band gap by doping, accompanied by large enhancements of the electronic specific-heat coefficient and magnetization [19,20,23,24]. Furthermore, weak doping often results in a magnetic instability [19,20,24,25]. For $\mathrm{FeGa}_{3}$, electron-type doping induces a crossover to a correlated metallic state at $x \approx 0.05$ and $y \approx 0.006$ in $\mathrm{Fe}_{1-x} \mathrm{Co}_{x} \mathrm{Ga}_{3}$ [34] and $\mathrm{FeGa}_{3-y} \mathrm{Ge}_{y}$ [35], respectively. Interestingly, further doping with $\mathrm{Ge}$ in $\mathrm{FeGa}_{3-y} \mathrm{Ge}_{y}$ leads to a ferromagnetic quantum critical point at $x \approx 0.016-0.05$ [35,37], whereas for $\mathrm{Fe}_{0.5} \mathrm{Co}_{0.5} \mathrm{Ga}_{3}$ nuclear spin-lattice relaxation measurements revealed a very fast relaxation with temperature dependence $1 / T_{1} \propto T^{1 / 2}$ being a unique feature of weakly and nearly antiferromagnetic metals [46]. A recent computational study suggested the formation of an itinerant ferromagnetic state with half-metallic properties in $\mathrm{FeGa}_{3}$ in case of doping with both electrons and holes [43]. Motivated by these results, we dope $\mathrm{FeGa}_{3}$ with holes on both $\mathrm{Fe}$ and $\mathrm{Ga}$ sites. We choose $\mathrm{Mn}$ and $\mathrm{Zn}$ as dopants because they lie in the periodic table next to iron and gallium, respectively, and have one less valence electron, corresponding to hole doping. We investigate the evolution in the thermodynamic and transport properties of single crystals of $\mathrm{Fe}_{1-x} \mathrm{Mn}_{x} \mathrm{Ga}_{3}$ and $\mathrm{FeGa}_{3-y} \mathrm{Zn}_{y}$ by means of magnetization, specific heat, electrical resistivity, and neutron powder diffraction measurements. We interpret the experimental results in the context of electronic-structure calculations based on DMFT.

\section{METHODS}

\section{A. Experiment ${ }^{1}$}

Single crystals of $\mathrm{FeGa}_{3}$ and $\mathrm{RuGa}_{3}$, as well as the series $\mathrm{Fe}_{1-x} \mathrm{Mn}_{x} \mathrm{Ga}_{3}(0<x<0.12)$ and $\mathrm{FeGa}_{3-y} \mathrm{Zn}_{y}(0<y<$ 0.06), were grown from gallium flux. Mixtures of high-purity elements in the ratio $\mathrm{Fe}: \mathrm{Ga}=1: 6.7-11.5, \mathrm{Ru}: \mathrm{Ga}=1: 15.7$, Fe:Mn:Ga $=\left(1-x_{\text {nom }}\right): x_{\text {nom }}: 8.7-9.3\left(0 \leqslant x_{\text {nom }}<70 \%\right)$ or Fe:Zn:Ga $=0.066: x_{\text {nom }}:\left(1-x_{\text {nom }}\right)\left(0 \leqslant x_{\text {nom }}<28.3 \%\right)$ were placed in $\mathrm{Al}_{2} \mathrm{O}_{3}$ crucibles and sealed in quartz ampoules under a low pressure of $\sim 200$ mbar of Ar gas. The ampoules were heated up to $1050^{\circ} \mathrm{C}$ and then slowly $\left(\sim 3^{\circ} \mathrm{C}\right.$ per hour $)$ cooled down to $650^{\circ} \mathrm{C}$ in case of undoped $\mathrm{FeGa}_{3}, \mathrm{RuGa}_{3}$, and $\mathrm{Zn}$-doped $\mathrm{FeGa}_{3}$, and to $550^{\circ} \mathrm{C}$ for $\mathrm{Mn}$-doped $\mathrm{FeGa}_{3}$, at which point the molten flux was separated from the crystals by spinning in a centrifuge. The quartz tubes were cracked only after cooling down to room temperature to prevent oxidation of crystals. The remaining flux was removed from crystal faces mechanically using latex sheets.

The microstructures of crystals were examined optically and with a scanning electron microscope (LEO/550) equipped with a Robinson backscatter detector. Chemical composition was determined by means of energy dispersive X-ray spectroscopy (EDXS) studies performed at several points across a crystal surface, based on integrated intensities of the $\mathrm{Fe} K$, $\mathrm{Mn} K, \mathrm{Ru} L$, and $\mathrm{Ga} L$ x-ray lines. The average composition for crystals of $\mathrm{FeGa}_{3}\left(\mathrm{Fe}_{0.97(1)} \mathrm{Ga}_{3.03(1)}\right)$ was taken as a standard to calculate the compositions of Mn- and Zn-doped crystals.

The measured concentrations of the dopants $\left(x_{\mathrm{m}}\right)$ increase gradually with the nominal doping level, and depend to some extent on the Fe:Ga ratio (Fig. 1). There is no indication for a limit of solubility of $\mathrm{Mn}$ or $\mathrm{Zn}$ in $\mathrm{FeGa}_{3}$, although we were not able to grow crystals via this approach with $x \geq 0.12$ and $y \gtrsim 0.06$. The compositions discussed here always refer to the measured doping levels.

${ }^{1}$ Certain commercial equipment, instruments, or materials are identified in this document. Such identification does not imply recommendation or endorsement by the National Institute of Standards and Technology nor does it imply that the products identified are necessarily the best available for the purpose.

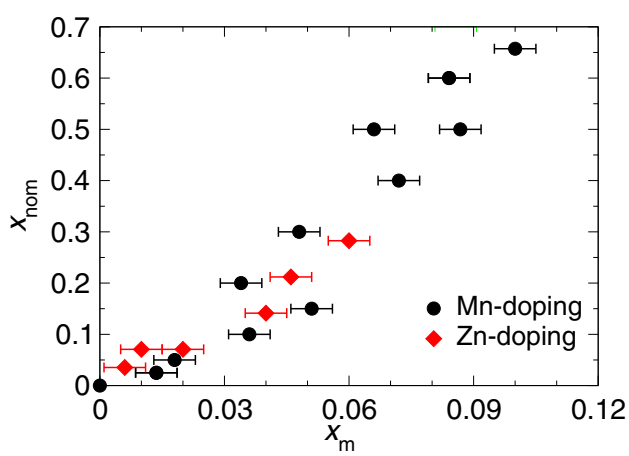

FIG. 1. (Color online) The relation between actual $\left(x_{\mathrm{m}}\right)$ and nominal $x_{\text {nom }}$ doping levels in $\mathrm{Fe}_{1-x} \mathrm{Mn}_{x} \mathrm{Ga}_{3}$ (black circles) and $\mathrm{FeGa}_{3-y} \mathrm{Zn}_{y}$ (red diamonds). Error bars indicate one standard deviation of the measured $x_{\mathrm{m}}$ values. 


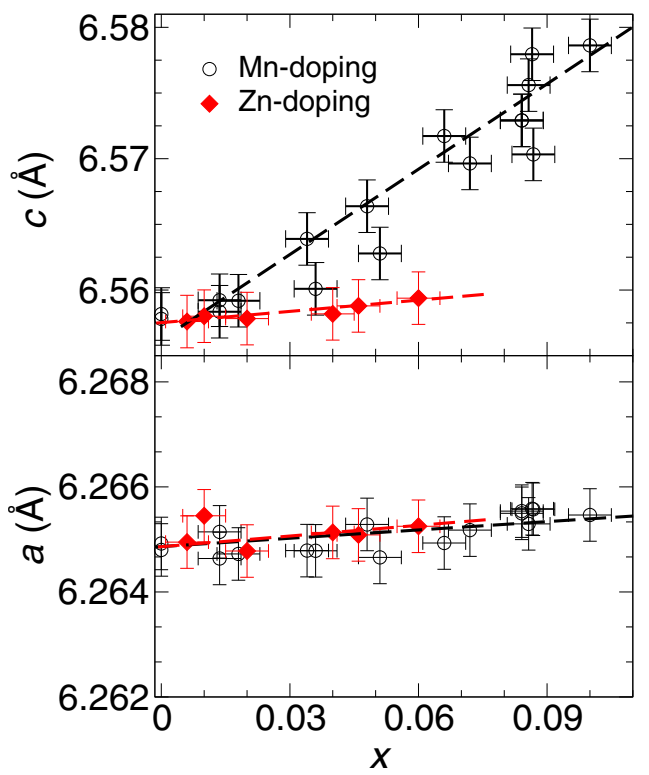

FIG. 2. (Color online) Lattice parameters $a$ and $c$ as functions of actual doping level $(x)$ for $\mathrm{Fe}_{1-x} \mathrm{Mn}_{x} \mathrm{Ga}_{3}$ (black circles) and $\mathrm{FeGa}_{3-y} \mathrm{Zn}_{y}$ (red diamonds). Dashed lines are guides for the eye.

Structural characterization at room temperature was carried out on powdered crystals using a Bruker D-8 advance diffractometer using $\mathrm{Cu} K \alpha$ radiation. Refinements of the recorded diffraction patterns were performed using the JANA2006 program [47]. The x-ray powder diffraction measurements confirmed that $\mathrm{FeGa}_{3}$ and $\mathrm{RuGa}_{3}$ form in a tetragonal structure with space group $P 42 / m n m$ (\#136) and lattice parameters that are in agreement with previous reports [32,41,48]. Doping of $\mathrm{FeGa}_{3}$ with $\mathrm{Mn}$ leads to a monotonic increase in lattice parameter $c$ with increasing $x$, following Vegard's law (Fig. 2). For doping with $\mathrm{Zn}$, lattice parameters vary only slightly, in line with a recent report on polycrystalline samples [49]. X-ray powder diffraction patterns of $\mathrm{Fe}_{1-x} \mathrm{Mn}_{x} \mathrm{Ga}_{3}(0<x<0.12)$ and $\mathrm{FeGa}_{3-y} \mathrm{Zn}_{y}(0<y<0.06)$ are all consistent with the $\mathrm{FeGa}_{3}$-type structure. No evidence for the presence of an impurity phase or for phase separation in the single crystals was found, apart from trace inclusions of gallium flux.

The magnetization studies in the temperature range between 1.8 and $400 \mathrm{~K}$ were carried out in a Quantum Design Magnetic Properties Measurement System (MPMS). At temperatures between 400 and $900 \mathrm{~K}$, the magnetization was studied using the Vibrating Sample Magnetometer (VSM) option of a Quantum Design Physical Property Measurement System (PPMS). The heat capacity was determined by the relaxation method at temperatures between 0.44 and $300 \mathrm{~K}$ using the PPMS. Both ac and dc electrical resistivity measurements were performed in the same system with a standard four-probe setup, where electrical contacts were made using silver-filled epoxy.

Neutron diffraction was measured on $\sim 6-\mathrm{g}$ powders of $\mathrm{FeGa}_{3}$ and $\mathrm{Fe}_{0.95} \mathrm{Mn}_{0.05} \mathrm{Ga}_{3}$ obtained by triturating single crystals. The diffraction measurements were carried out at temperatures ranging from 1.5 to $300 \mathrm{~K}$ using the High Resolution Powder Diffractometer BT-1 of the NIST Center for Neutron Research, with the $\mathrm{Ge}(311)$ monochromator giving neutrons with wavelength $\lambda=2.079 \AA$. The diffraction patterns were remeasured in a different cryostat to verify that all the observed diffraction peaks originate with the sample. The Rietveld refinement of the nuclear structure was done with the program FULLPROF [50].

\section{B. Computation}

The combination of dynamical mean field theory (DMFT) and density functional theory (DFT) in the local density approximation (LDA) was used to compute spectral, magnetic, and transport properties of pure and hole-doped $\mathrm{FeGa}_{3}$. For the DFT part, we employed WIEN2K [51]. The DMFT [52] impurity problem was solved using a hybridization expansion continuous time quantum Monte Carlo (CTQMC) method [53,54]. We use a projection-based DFT+DMFT setup with full charge self-consistency [55]. For $\mathrm{FeGa}_{3}$, we use a Hubbard interaction $U=5.0 \mathrm{eV}$ and a Hund's rule coupling $J=0.7 \mathrm{eV}$ for the $3 d$ states of iron. These values were found to be appropriate for iron-based compounds $[10,56]$. For the $4 d$-electron compound $\mathrm{RuGa}_{3}$, we use $U=2.5 \mathrm{eV}$ and $J=0.4 \mathrm{eV}$ [57]. Response functions are obtained within the Kubo formalism [12]. The doping is simulated through the virtual crystal approximation (VCA).

\section{RESULTS}

\section{A. $\mathrm{FeGa}_{3}$}

Figure 3 shows the dc magnetic susceptibility $\chi=M / H$ of $\mathrm{FeGa}_{3}$ and of its isostructural and isoelectronic homolog $\mathrm{RuGa}_{3}$, measured with a magnetic field $H=10 \mathrm{kOe}$ applied parallel to the $a b$ plane, and along the $c$ axis of the crystal. Overall, $\chi(T)$ for $\mathrm{FeGa}_{3}$ is similar to that reported previously for a set of randomly oriented crystals over a more limited temperature range $[32,39]$. The magnetic anisotropy is mostly reflected in a temperature-independent offset between values

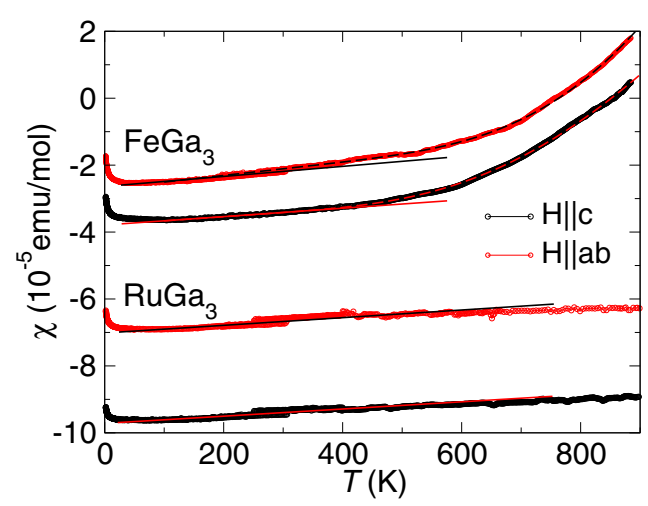

FIG. 3. (Color online) Magnetic susceptibility of single crystals of $\mathrm{FeGa}_{3}$ and $\mathrm{RuGa}_{3}$ measured in a magnetic field of $10 \mathrm{kOe}$ applied in the tetragonal $a b$ plane (red circles) or along the $c$ axis (black circles). Thin solid lines indicate linear terms $a T$ in $\chi(T)$ for $\mathrm{FeGa}_{3}$ with $a=1.3 \times 10^{-8} \mathrm{emu} /(\mathrm{K} \mathrm{mol})$ and $a=1.9 \times 10^{-8} \mathrm{emu} /(\mathrm{K}$ mol) for $H \| a b$ and $H \| c$, respectively, and for $\mathrm{RuGa}_{3}$ with $a=0.7 \times 10^{-8} \mathrm{emu} /(\mathrm{K} \mathrm{mol})$ independent of the field direction. Dashed lines represent fits to the activated temperature dependence $\chi(T) \propto \exp \left[-\Delta_{\mathrm{S}} /\left(\mathrm{k}_{\mathrm{B}} T\right)\right]$ at $T \gtrsim 500 \mathrm{~K}$. 
obtained when the magnetic field is applied perpendicular or parallel to the $c$ axis in the tetragonal crystal lattice, whereas the temperature dependence is almost the same in both directions. An even larger magnetic anisotropy was found for $\mathrm{RuGa}_{3}$.

For both $\mathrm{FeGa}_{3}$ and $\mathrm{RuGa}_{3}, \chi$ is negative at low temperatures and increases nearly linearly with increasing temperature up to $\sim 500 \mathrm{~K}$. Slight Curie tails in $\chi(T)$ at the lowest temperatures (Fig. 3) may be assigned to a small number $\sim 0.06 \%$ of $S=\frac{1}{2}$ paramagnetic impurities. After correction for these impurities, an extrapolation of $\chi(T)$ to $T=0 \mathrm{~K}$ results in $\chi_{0(a b)} \approx-26 \times 10^{-6} \mathrm{emu} \mathrm{mol}^{-1}$ and $\chi_{0(c)} \approx-38 \times 10^{-6}$ emu $\mathrm{mol}^{-1}$ for $\mathrm{FeGa}_{3}$ and $\chi_{0(a b)} \approx$ $-70 \times 10^{-6} \mathrm{emu} \mathrm{mol}^{-1}, \chi_{0(c)} \approx-94 \times 10^{-6} \mathrm{emu} \mathrm{mol}^{-1}$ for $\mathrm{RuGa}_{3}$. The diamagnetism of the closed-shell ions [58] gives the values of $-37 \times 10^{-6}$ emu mol $^{-1}$ and $-47 \times$ $10^{-6}$ emu mol $^{-1}$ for $\mathrm{FeGa}_{3}$ and $\mathrm{RuGa}_{3}$, respectively, which are comparable with the measured $\chi_{0}$ values. The lattice diamagnetism resulting from the interband effect of the magnetic field [59] is likely also responsible for the observed magnetic anisotropies.

The almost linear increase of the magnetic susceptibilities with increasing temperature for both $\mathrm{FeGa}_{3}$ and $\mathrm{RuGa}_{3}$ observed in broad temperature ranges (see Fig. 3) can be assigned to a slight shrinking of their band gaps. In semiconductors, electron-phonon interactions usually lead to a shrinking of the gap with increasing temperature due to an increase in the phonon population [60]. This, in turn, leads to a small and gradual increase of the magnetic susceptibility [61].

The magnetic susceptibility of $\mathrm{RuGa}_{3}$ remains negative and only weakly temperature dependent up to the highest temperatures. In contrast, for $\mathrm{FeGa}_{3}$ the slope of $\chi(T)$ starts to increase significantly at temperatures above $\sim 500 \mathrm{~K}$. The temperature dependencies of the magnetic susceptibilities can be well described as $\chi(T) \propto \exp \left[-\Delta_{\mathrm{S}} /\left(\mathrm{k}_{\mathrm{B}} T\right)\right]$. Here, $k_{\mathrm{B}}$ denotes the Boltzmann constant. The spin gaps derived from such fits are $\Delta_{\mathrm{S}-\mathrm{ab}}=0.33 \mathrm{eV}$ for $H \| a b$ and $\Delta_{\mathrm{S}-\mathrm{c}}=0.41 \mathrm{eV}$ for $H \| c$.

The most direct information about the magnetic state of $\mathrm{FeGa}_{3}$ can be gained from neutron diffraction measurements. Figure 4 shows a neutron powder diffraction pattern collected at a temperature of $1.5 \mathrm{~K}$. Rietveld refinement of the nuclear structure confirmed the tetragonal symmetry with space group $P 4_{2} / m n m$. Structural parameters derived from the refinements of the neutron diffraction patterns measured at temperatures of 1.5 and $300 \mathrm{~K}$ are presented in Table I. The results obtained at room temperature are in excellent agreement with the structural data attained based on our $\mathrm{X}$-ray powder diffraction patterns (Table I) and with previous reports $[32,41,48]$.

In addition to nuclear reflections, there are several clearly discernible extra peaks in the neutron patterns. The strongest additional peaks are at wave vectors $Q=0.986 \AA^{-1}$, $1.058 \AA^{-1}, 1.478 \AA^{-1}$, and $1.586 \AA^{-1}$ and are marked in the inset of Fig. 4. The absence of diffraction peaks at similar values of $Q$ in room temperature x-ray powder diffraction patterns from the same sample, measured before and after collecting the neutron diffraction data, strongly argues for a magnetic origin of the additional peaks. The emergence of the extra peaks only at rather small values of $Q$ provides further support for their magnetic character because the magnetic form factor falls off quite rapidly with diffraction angle. The magnetic peaks are almost identical at temperatures of 1.5 and $300 \mathrm{~K}$ and their $Q$ widths are resolution limited, thus indicating long-range magnetic order. This advocates that $\mathrm{FeGa}_{3}$ is an antiferromagnet with the ordering temperature $T_{N}$ above $300 \mathrm{~K}$. The presence of distinct magnetic peaks in the collected neutron patterns implies that the size of the staggered moments is well above $0.1 \mu_{B}$, which is the approximate detection limit of the neutron powder diffraction method. Simultaneously, the small size of the magnetic peaks compared to the nuclear peaks makes magnetic moments larger than $1-1.5 \mu_{\mathrm{B}}$ per $\mathrm{Fe}$ very unlikely.

The magnetic peaks can be indexed only by a propagation vector that is incommensurate with the crystal lattice in at least one direction. The best description of the observed peaks was achieved assuming $\mathbf{k}=(0.5,0.25,0.44)$, indicating that the tetragonal symmetry of the nuclear structure is not shared by the magnetic structure. A complete solution of this complex magnetic structure requires further experimental studies, in particular, neutron diffraction measurements of single crystals.

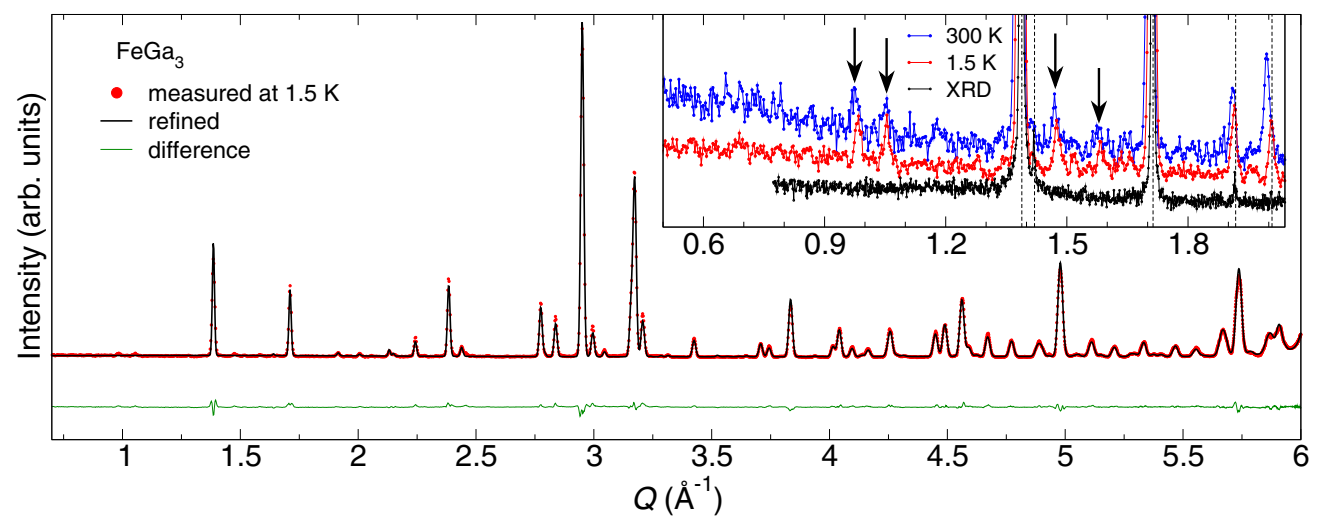

FIG. 4. (Color online) Results of neutron powder diffraction experiments for FeGa $\mathrm{F}_{3}$. The main panel shows the Rietveld refinement (black) of the nuclear structure based on the neutron diffraction pattern (red) collected at $1.5 \mathrm{~K}$. The difference curve is shown in green. The inset shows the low-angle range of the neutron diffraction patterns measured at $1.5 \mathrm{~K}$ (red) and at room temperature (blue), together with the x-ray powder diffraction data measured at $300 \mathrm{~K}$ on the same powder. Dashed vertical lines show the positions of nuclear peaks. The main magnetic peaks are indicated by arrows. 
TABLE I. Structural parameters derived from the Rietveld refinements of the nuclear structure based on our neutron powder diffraction patterns measured for $\mathrm{FeGa}_{3}$ at temperatures of 1.5 and $300 \mathrm{~K}$. The last columns present results of the Rietveld refinement of the x-ray powder diffraction (XRD) pattern for $\mathrm{FeGa}_{3}$ collected at room temperature. All these refinements resulted in full occupancies of the three atomic sites.

\begin{tabular}{|c|c|c|c|c|c|c|c|c|c|c|c|}
\hline \multicolumn{2}{|c|}{ Lattice parameters } & \multicolumn{3}{|c|}{$T=1.5 \mathrm{~K}$} & \multicolumn{3}{|c|}{$T=300 \mathrm{~K}$} & \multicolumn{4}{|c|}{$\mathrm{XRD} ; T=295 \mathrm{~K}$} \\
\hline & $a(\AA)$ & & $6.2528(2)$ & & & $6.2645(9)$ & & & 6.265 & $2(1)$ & \\
\hline & $c(\AA)$ & & $6.5462(8)$ & & & $6.5590(15)$ & & & 6.558 & $6(1)$ & \\
\hline Atom & Wyckoff site & $x$ & $y$ & $z$ & $x$ & $y$ & $z$ & $x$ & $y$ & $z$ & $B_{\text {iso }}$ \\
\hline $\mathrm{Fe}$ & $4 f$ & $0.1566(3)$ & $0.1566(3)$ & 0 & $0.1552(8)$ & $0.1552(8)$ & 0 & $0.15739(6)$ & $0.15739(6)$ & 0 & $0.054(1)$ \\
\hline Ga1 & $8 j$ & $0.3448(2)$ & $0.3448(2)$ & $0.2635(2)$ & $0.3445(9)$ & $0.3445(9)$ & $0.2626(10)$ & $0.34462(5)$ & $0.34462(5)$ & $0.26193(7)$ & $0.045(2)$ \\
\hline $\mathrm{Ga} 2$ & $4 c$ & 0 & 0.5 & 0 & 0 & 0.5 & 0 & 0 & 0.5 & 0 & $0.046(1)$ \\
\hline
\end{tabular}

The overall temperature dependence of the specific heat $C_{p}$ for $\mathrm{FeGa}_{3}$ is depicted in Fig. 5(a) and is similar to that reported by Hadano et al. [32]. At ambient temperature, the specific heat nearly reaches $100 \mathrm{~J} /(\mathrm{mol} \mathrm{K})$, which is the result expected from the Dulong-Petit law. At $T<3 \mathrm{~K}$, the experimental data follow the dependence anticipated for the contribution of phonons $C_{p}(T)=\beta T^{3}$ [see inset in Fig. 5(b)] with $\beta \approx 0.139 \mathrm{~mJ} /\left(\mathrm{mol} \mathrm{K}{ }^{4}\right)$. The Debye model gives $\beta=$ $(12 / 5) R n \pi^{4} \theta_{\mathrm{D}}^{-3}$, where $n$ is the number of atoms per formula unit and $R$ is the gas constant. Thus, we estimate a Debye temperature $\theta_{\mathrm{D}} \approx 382 \mathrm{~K}$.

For $\mathrm{FeGa}_{3}$, the magnetic contribution to the specific heat is expected to be negligible at temperatures below $\sim 300 \mathrm{~K}$ since the magnetic susceptibility is diamagnetic and only weakly temperature dependent in this regime. Consequently, at temperatures above $\sim 3 \mathrm{~K}, C_{p}(T)$ is strongly dominated by the vibrational properties of the $\mathrm{Fe}$ and $\mathrm{Ga}$ sublattices of $\mathrm{FeGa}_{3}$. Attempts to fit the overall temperature dependence of the specific heat as the lattice specific heat $C_{\text {lattice }}(T)$ given by a Debye model are not satisfactory [Fig. 5(a)]. In order to get an acceptable description of the experimental $C_{p}(T)$, the lattice specific heat of $\mathrm{FeGa}_{3}$ has been modeled by the sum of contributions from Debye-type $\left(C_{\mathrm{D}}\right)$ and Einstein-type $\left(C_{\mathrm{E}}\right)$ modes, using the following expressions:

$$
C_{\mathrm{D}}(T)=R n_{\mathrm{D}} \int_{0}^{\Theta_{\mathrm{D}} / T} \frac{x^{4} e^{x}}{\left(e^{x}-1\right)^{2}} d x
$$

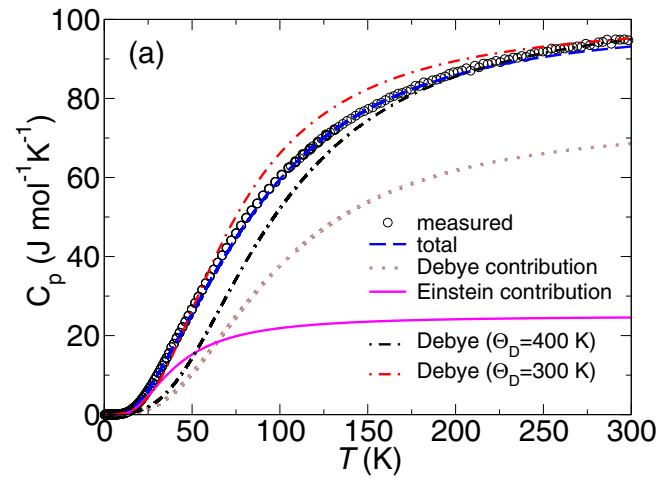

and

$$
C_{\mathrm{E}}(T)=R n_{\mathrm{E}}\left(\frac{\Theta_{\mathrm{E}}}{T}\right)^{2} \frac{e^{\frac{\Theta_{\mathrm{E}}}{T}}}{\left(e^{\frac{\Theta_{\mathrm{E}}}{T}}-1\right)^{2}},
$$

where $n_{\mathrm{D}}$ and $n_{\mathrm{E}}$ denote the number of Debye and Einstein modes per formula unit of $\mathrm{FeGa}_{3}$.

The best match with the experimental data is achieved by assuming $n_{\mathrm{D}}=9$ Debye-type modes with $\theta_{\mathrm{D}}=400 \mathrm{~K}$, one Einstein-type mode with a characteristic temperature $\theta_{\mathrm{E}}=94 \mathrm{~K}$ and two Einstein-type modes with $\theta_{\mathrm{E}}=140 \mathrm{~K}$, resulting in a total of 12 phonon modes, as expected for $\mathrm{FeGa}_{3}$. In Fig. 5(b), we plot $C_{p} / T^{3}$ versus $T$. The clear maximum indicates that Einstein modes must be introduced for a reasonable fit.

The presence of the Einstein modes in $\mathrm{FeGa}_{3}$ is rather unexpected. Generally, low-energy optical modes are associated with specific structure types. For compounds with cagelike crystal structures, for instance, the Einstein term in the specific heat reflects local vibrations of loosely bound atoms inside the cages. Yet, in $\mathrm{FeGa}_{3}$ each atom has several neighbors at distances shorter than the sum of adjacent nominal atomic radii [48]. Single-crystal x-ray diffraction measurements do not show an enhancement of any displacement parameters in $\mathrm{FeGa}_{3}$ [41] that could otherwise indicate the presence of loosely bound atoms in the crystal lattice. Therefore, the evidence in the specific heat of $\mathrm{FeGa}_{3}$ for local vibrations calls for further investigations, such as thermal expansion measurements.

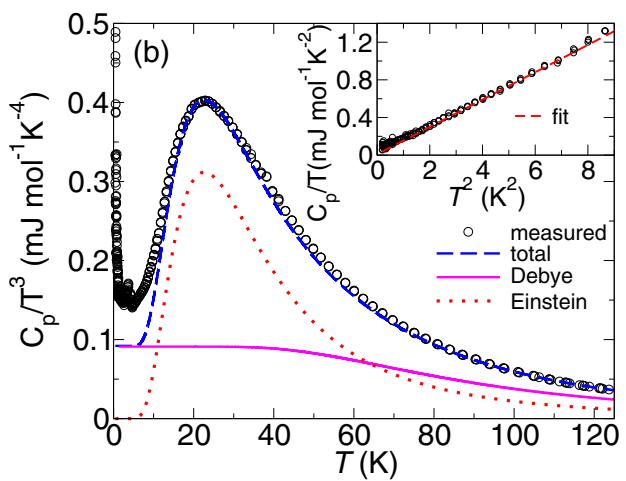

FIG. 5. (Color online) Specific heat $C_{p}$ of $\mathrm{FeGa}_{3}$ (black circles) modeled with a series of Einstein-type (solid line) and Debye-type (dotted line) lattice terms (see text). The blue dashed line represents the best fit of the assumed model to the experimental data. Dashed-dotted thin lines in panel (a) represent the "total" specific heat calculated based on the Debye model, assuming Debye temperatures of $300 \mathrm{~K}$ (red) and $400 \mathrm{~K}$ (black). A maximum in $C / T^{3}(T)$ dependence shown in panel (b) is the hallmark of the presence of Einstein modes. The inset in panel (b) shows $C_{p} / T$ at lowest temperatures plotted versus $T^{2}$. The red dashed line represents the fit to the experimental data using $C_{p} / T\left(T^{2}\right)=\beta T^{2}$. 

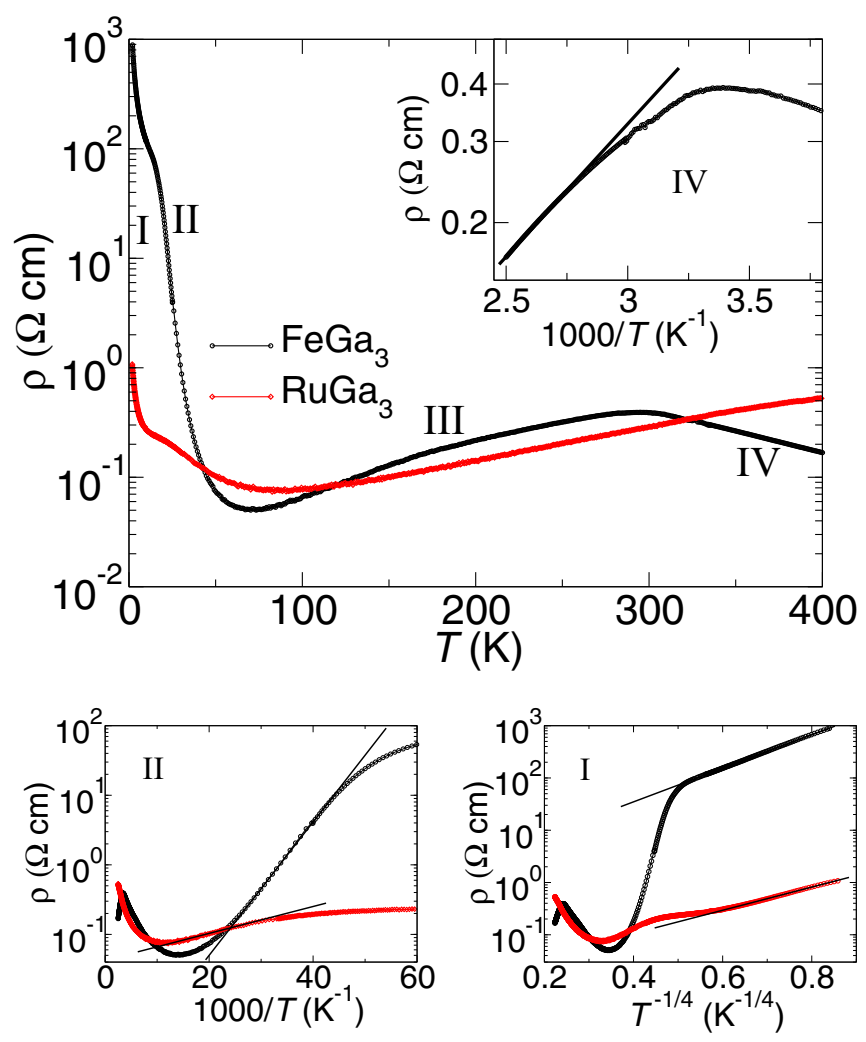

FIG. 6. (Color online) Electrical resistivity $\rho$ for $\mathrm{FeGa}_{3}$ (black circles) and $\mathrm{RuGa}_{3}$ (red circles). Roman numbers I-IV denote four temperature regimes as described in the text. The inset shows the resistivity for $\mathrm{FeGa}_{3}$ in the temperature range 260-400 K (IV) on a logarithmic scale as a function of inverse temperature. The solid line represents the fit of the experimental data with Arrhenius law $\rho(T)=\rho_{0}(T) \exp \left[E_{\mathrm{g}} /\left(2 k_{\mathrm{B}} T\right)\right]$, giving the activation gap $E_{\mathrm{g}}=0.4 \mathrm{eV}$. The left small panel displays Arrhenius plots for the range II with solid lines indicating the best fits of the resistivity data to the Arrhenius expression $\rho(T)=\rho_{0}(T) \exp \left[E_{\mathrm{d}} /\left(2 k_{\mathrm{B}} T\right)\right]$ at $24 \mathrm{~K}<T<44 \mathrm{~K}$ and $31 \mathrm{~K}<T<83 \mathrm{~K}$, resulting in activation energies of $E_{\mathrm{d}} \approx 40$ and $20 \mathrm{meV}$ for $\mathrm{FeGa}_{3}$ and $\mathrm{RuGa}_{3}$, respectively. The right small panel shows the resistivities at the lowest temperatures (I) plotted versus $T^{-1 / 4}$ to display the 3D VRH conduction among in-gap states. Solid lines indicate the best linear regression fits.

The electrical resistivity $\rho$ was measured on single crystals of $\mathrm{FeGa}_{3}$ and $\mathrm{RuGa}_{3}$ (Fig. 6). The obtained $\rho(T)$ for $\mathrm{FeGa}_{3}$ is very similar to that found in previous reports based on single crystals $[32,41]$, and differs notably from some results for polycrystalline specimens $[42,62]$. In turn, for $\mathrm{RuGa}_{3}$, there are substantial differences between our resistivity data and the $\rho(T)$ curves reported previously for both single crystals [41] and polycrystalline specimens $[37,42]$. Such a sensitivity of physical properties to structure, morphology, and chemical composition is typical for correlated insulators.

The absolute values of the resistivity are similar for crystals of $\mathrm{FeGa}_{3}$ and $\mathrm{RuGa}_{3}$ grown from Ga flux. The resistivity of $\mathrm{FeGa}_{3}$ displays four distinct temperature regimes, which we indicate by roman numbers (see Fig. 6): At $T>350 \mathrm{~K}$ (regime IV), the resistivity of $\mathrm{FeGa}_{3}$ follows the Arrhenius law $\rho(T) / \rho_{0}(T)=\exp \left[E_{\mathrm{g}} /\left(2 k_{\mathrm{B}} T\right)\right]$, with an activation energy gap $E_{\mathrm{g}} \approx 0.4 \mathrm{eV}$ [Fig. 6(b)], in line with the size of the band gap derived from a spectroscopic study and electronic-structure calculations [31,33,41]. Below $T \sim 300 \mathrm{~K}$ (III), the resistivity of $\mathrm{FeGa}_{3}$ decreases with decreasing temperature. For $\mathrm{RuGa}_{3}$, a similar behavior in resistivity is found to extend over an even broader temperature range. Since in $\mathrm{RuGa}_{3}$ the orbitals responsible for low-energy excitations are of $4 d$ character that are much more delocalized than their $3 d$ counterparts in $\mathrm{FeGa}_{3}$, the emergence of the metallic-type range (III) in the resistivity of $\mathrm{FeGa}_{3}$ is likely not associated directly with correlation effects. At lower temperatures, the resistivities of both $\mathrm{FeGa}_{3}$ and $\mathrm{RuGa}_{3}$ increase again. The temperature dependencies of the resistivity from 20 to $45 \mathrm{~K}$ for $\mathrm{FeGa}_{3}$ and from 30 to $80 \mathrm{~K}$ for $\mathrm{RuGa}_{3}$ [II, Fig. 6(c)] can be again well described by the Arrhenius law $\rho(T) / \rho_{0}(T)=\exp \left[E_{\mathrm{d}} /\left(2 k_{\mathrm{B}} T\right)\right]$, with activation energies $E_{\mathrm{d}} \approx 40$ and $20 \mathrm{meV}$ for $\mathrm{FeGa}_{3}$ and $\mathrm{RuGa}_{3}$, respectively. These activation gaps are more than one order of magnitude smaller than the band gaps for both compounds [31,40,41]. Finally, at the lowest temperatures (I), the resistivities of both $\mathrm{FeGa}_{3}$ and $\mathrm{RuGa}_{3}$ vary as $\rho(T) \propto$ $\left.\exp \left[\left(T_{\mathrm{M}} / T\right)^{1 / 4}\right)\right]$ [Fig. 6(d)]. Such a temperature dependence is predicted for the conduction due to variable range hopping (VRH) among localized in-gap states in a three-dimensional (3D) system, a dominant conduction mechanism in various classes of lightly doped semiconductors at low temperatures. Although the character of the temperature dependence of the resistivity in this temperature range was similar for several studied crystals of both $\mathrm{FeGa}_{3}$ and $\mathrm{RuGa}_{3}$, considerable variations in values of $T_{\mathrm{M}}$ were observed.

The temperature dependencies of the resistivities for $\mathrm{FeGa}_{3}$ and $\mathrm{RuGa}_{3}$ observed in regimes I-III can be ascribed to the presence of numerous localized donor-type states in their band gaps. Indeed, a recent nuclear spin-lattice relaxation study for $\mathrm{FeGa}_{3}$ unambiguously proved the existence of in-gap states located just below the bottom of the conduction band [46]. The number of these states estimated from the Hall constant [32] is $\sim 1.6 \times 10^{16} \mathrm{~cm}^{-3}$, which is much larger than typical impurity levels observed in intrinsic band semiconductors (e.g., Si, Ge) and even in correlated band insulators (e.g., FeSi, FeSb ) $^{\text {[46] }}$ Measurements of the Hall effect performed by Hadano et al. [32] indicated that in region III mobility of carriers decreases strongly with increasing temperature $\left(\mu \sim T^{-5 / 2}\right)$, whereas the number of carriers remains nearly unchanged. Consequently, the emergence of the metallic behavior of the resistivity can be explained as due to this strong decrease in carrier mobility in the saturation range, where electrons from all the donor levels are thermally excited to the conduction band. In turn, a gradual freezing of these donors leads to the activated $\rho(T)$ at temperatures below $\sim 45 \mathrm{~K}$ and $\sim 80 \mathrm{~K}$ for $\mathrm{FeGa}_{3}$ and $\mathrm{RuGa}_{3}$, respectively. Further study is needed to elucidate the origin and character of these numerous in-gap states.

\section{B. $\mathrm{Fe}_{1-x} \mathrm{Mn}_{x} \mathrm{Ga}_{3}$ and $\mathrm{FeGa}_{3-y} \mathrm{Zn}_{y}$}

Doping $\mathrm{FeGa}_{3}$ with both $\mathrm{Mn}$ and $\mathrm{Zn}$ results in drastic changes in $\rho(T)$, compared to undoped FeGa . Figure 7 presents the electrical resistivity of crystals doped with $\mathrm{Zn}$ [Fig. 7(a)] and with Mn [Fig. 7(b)]. Already for the lowest doping levels, the metallic range in $\rho(T)$ that was observed for undoped crystals of $\mathrm{FeGa}_{3}$ from $\sim 60$ to $\sim 300 \mathrm{~K}$ gives way 

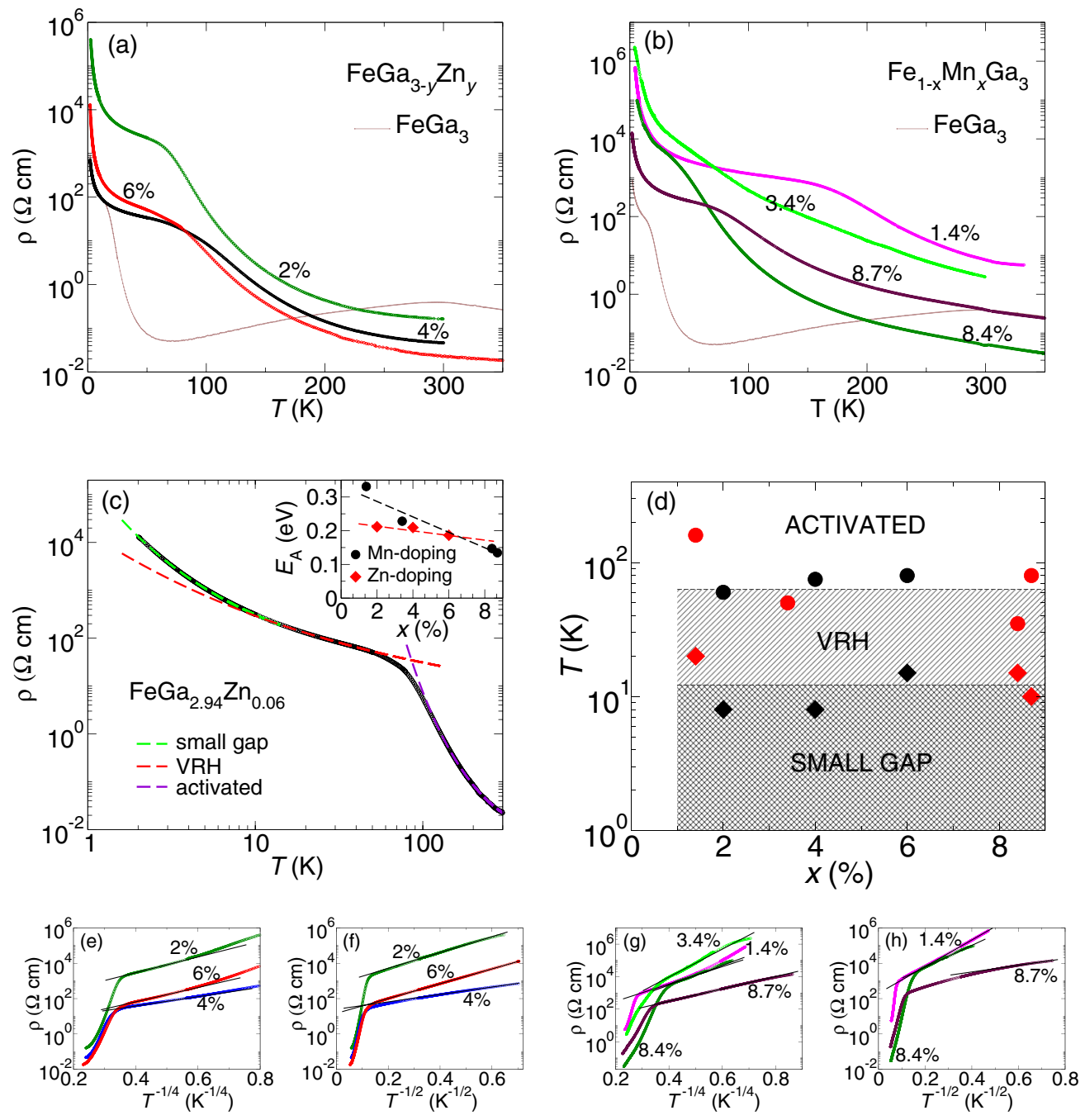

FIG. 7. (Color online) Electrical resistivity for $\mathrm{FeGa}_{3-y} \mathrm{Zn}_{y}(y=0.02 ; 0.04 ; 0.06)$ (a) and $\mathrm{Fe}_{1-x} \mathrm{Mn}_{x} \mathrm{Ga}_{3}(x=0.014 ; 0.034 ; 0.084 ; 0.087)$ (b) plotted together with the data for $\mathrm{FeGa}_{3}$ (brown solid lines). Panel (c) presents the resistivity of $\mathrm{FeGa}_{2.94} \mathrm{Zn}_{0.06}($ black circles) together with fits using Arrhenius law $\rho(T) \propto \exp \left[E_{\mathrm{d}} /\left(2 k_{\mathrm{B}} T\right)\right]$ for $T \gtrsim 100 \mathrm{~K}$ (dashed violet line), 3D VRH conduction $\rho(T) \propto \exp \left[\left(T_{\mathrm{M}} / T\right)^{1 / 4}\right]$ at $10 \mathrm{~K} \gtrsim T \gtrsim 50 \mathrm{~K}$ (dashed blue line), and based on the formula $\rho(T) \propto \exp \left[\left(T_{M} / T\right)^{1 / 2}\right]$ (dashed red line) describing 3D VRH conduction of the Efros-Shklovskii type [63] associated with the opening of a small gap within the doping induced density of states at $T \gtrsim 10 \mathrm{~K}$. The temperature ranges in which the resistivities of $\mathrm{FeGa}_{3-y} \mathrm{Zn}_{y}$ and $\mathrm{Fe}_{1-x} \mathrm{Mn}_{x} \mathrm{Ga}_{3}$ follow the described temperature dependencies are indicated in panel (d). The points denote crossover temperatures estimated as the midpoints between the corresponding temperature ranges. The resistivities of $\mathrm{FeGa}_{3-y} \mathrm{Zn}_{y}$ and $\mathrm{Fe}_{1-x} \mathrm{Mn}_{x} \mathrm{Ga}_{3}$ for different $x$ and $y$ are plotted versus $T^{-1 / 4}$ in panels (e) and (g), respectively, and as functions of $T^{-1 / 2}$ in panels (f) and (h), respectively. Solid lines in panels (e), (f), (g), and (h) indicate the best regression fits. The inset in panel (c) shows values of the average activation gap obtained from the fits to the Arrhenius expression at highest temperatures as a function of the actual doping level $x$.

to an activated temperature dependence followed by broad maxima at temperatures of $30-150 \mathrm{~K}$. Although qualitatively similar behaviors of the resistivities were observed for different crystals, the details of the shape and position of the maxima in $\rho(T)$ were found to be sample dependent, even for crystals within a given batch, where EDX microanalysis indicated the same chemical composition and a homogeneous distribution of the dopant atoms across the crystals.

Since the shape of the $\rho(T)$ for $\mathrm{FeGa}_{3}$ was assigned to the presence of impurity donor-type states inside the band gap $[32,46]$, the drastic change in $\rho(T)$ upon doping likely results from a compensation mechanism. Our results suggest that the number of holes introduced by doping almost immediately exceeds the number of impurity donor states present in the nominally undoped $\mathrm{FeGa}_{3}$. Consequently, the presence of localized acceptor-type states in the band gap may determine $\rho(T)$ for both $\mathrm{Fe}_{1-x} \mathrm{Mn}_{x} \mathrm{Ga}_{3}$ and $\mathrm{FeGa}_{3-y} \mathrm{Zn}_{y}$, at least at temperatures up to $\sim 400 \mathrm{~K}$. This is in line with $p$-type conduction found by means of thermopower measurements for polycrystals of $\mathrm{FeGa}_{2.97} \mathrm{Zn}_{0.03}$ and $\mathrm{FeGa}_{2.94} \mathrm{Zn}_{0.06}$ at temperatures until 400 and $500 \mathrm{~K}$, respectively [49].

We notice that there are three distinct temperature ranges in the resistivities of both series of compounds $\mathrm{Fe}_{1-x} \mathrm{Mn}_{x} \mathrm{Ga}_{3}$ and $\mathrm{FeGa}_{3-y} \mathrm{Zn}_{y}$, as indicated in Fig. 7(c). At high temperatures, the resistivity is activated. Application of the Arrhenius law to fit the resistivity data of the doped crystals leads to values of the 

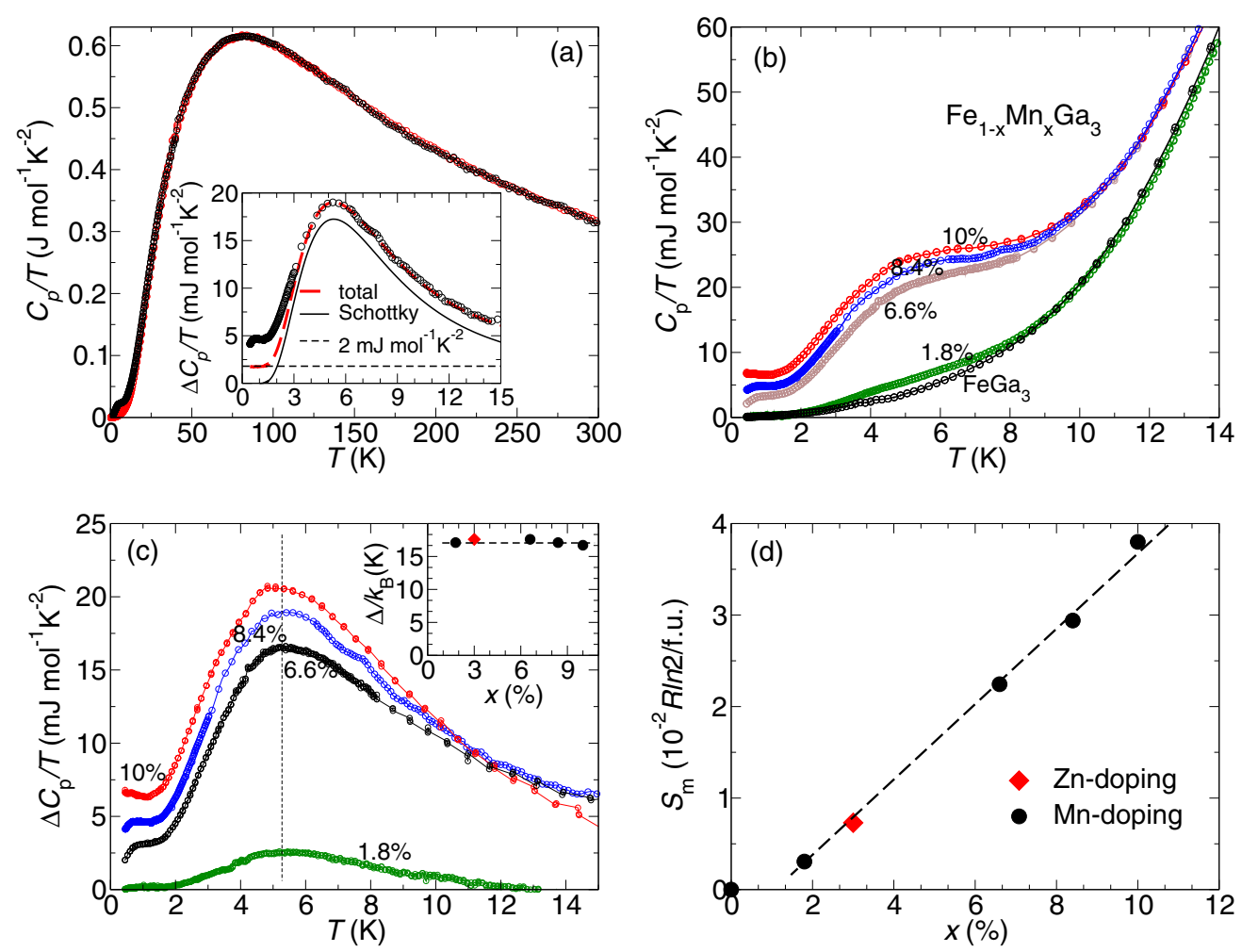

FIG. 8. (Color online) (a) Specific heat $C_{p} / T$ of $\mathrm{Fe}_{0.92} \mathrm{Mn}_{0.08} \mathrm{Ga}_{3}$ (black circles) together with the data of FeGa $\mathrm{Ga}_{3}$ (red squares). The inset displays the low-temperature magnetic specific heat, with a fit of data based on the two-level Schottky model as described in the text. (b) Low temperature $C_{p} / T(T)$ for the series of $\mathrm{Mn}$-doped $\mathrm{FeGa}_{3}$ together with the data for undoped $\mathrm{FeGa}_{3}$ (black empty circles). (c) $\Delta C_{p} / T(T)$ for the series of $\mathrm{Fe}_{1-x} \mathrm{Mn}_{x} \mathrm{Ga}_{3}(x=0 ; 1.8 \% ; 6.6 \% ; 8.4 \% ; 10 \%)$ estimated as described in the text. The thin dashed vertical line indicates the position of the maxima in $\Delta C_{p} / T(T)$. The inset shows the values of the energy gap $\Delta$ derived from the fits based on the two-level Schottky model. (d) Values of the magnetic entropy at a temperature of $14 \mathrm{~K}$ for various doping levels of Mn (black circles) and $\mathrm{Zn}$ (red square). The dashed line corresponds to the entropy of $0.4 R \ln 2$ per dopant. The dashed lines are guides for the eye.

activation gap $E_{\mathrm{A}}$ presented in the inset of Fig. 7(c). For both dopants, the average energy for these excitations decreases slightly with increasing doping level.

At lower temperatures, the resistivity is dominated by VRH among the localized in-gap states. The temperature dependence of the resistivity can be well described as

$$
\rho(T) \propto \exp \left[\left(T_{\mathrm{M}} / T\right)^{p}\right]
$$

The value of the exponent $p$ in Eq. (3) distinguishes different conduction mechanisms. For temperatures down to $\sim 10-15 \mathrm{~K}$, $p \approx 0.25$ [see Figs. 7(e) and 7(g)] as expected for the Mott's type VRH in a 3D semiconductor and also seen in nominally undoped $\mathrm{FeGa}_{3}$. At the lowest temperatures, the resistivity of all the doped crystals becomes larger than predicted by Mott's expression [see Fig. 7(c)]. Such an increase in the resistivity hints at the opening of a small gap within the doping-induced density of states at the Fermi level. Indeed, a good description of the experimental data for $\mathrm{FeGa}_{3-y} \mathrm{Zn}_{y}$ at temperatures below $\sim 10 \mathrm{~K}$ was achieved assuming $p=0.5$ [see Fig. 7(f)], as predicted for the 3D VRH conduction of the Efros-Shklovskii type [63]. For $\mathrm{Fe}_{1-x} \mathrm{Mn}_{x} \mathrm{Ga}_{3}$, the values of the exponent $p$ for the resistivity at lowest temperatures are somewhat ambiguous. Attempts to describe the experimental data assuming $p=0.5$ are shown in Fig. 7(h). The temperature ranges on which the electrical resistivities of $\mathrm{FeGa}_{3-y} \mathrm{Zn}_{y}$ and
$\mathrm{Fe}_{1-x} \mathrm{Mn}_{x} \mathrm{Ga}_{3}$ follow the three distinct temperature dependencies are indicated in Fig. 7(d).

Figure 8(a) compares $C_{p} / T$ as a function of temperature for $\mathrm{Fe}_{0.92} \mathrm{Mn}_{0.08} \mathrm{Ga}_{3}$ to the data for undoped $\mathrm{FeGa}_{3}$. The specific heat does not give any indication for a phase transition in either compound. Overall, the specific heat for the doped crystal is very similar to that of $\mathrm{FeGa}_{3}$, indicating that their phonon densities of states are very much alike. However, there is an additional contribution to $C_{p}$, that is present in the doped sample for $T<20 \mathrm{~K}$. In order to isolate it, we subtracted from the total specific heat of $\mathrm{Fe}_{0.92} \mathrm{Mn}_{0.08} \mathrm{Ga}_{3}$ the corresponding data for undoped $\mathrm{FeGa}_{3}$, taking advantage of their similar lattice specific heats and the negligible electronic contribution for insulating $\mathrm{FeGa}_{3}$. The resulting $\Delta C_{p}(T)=C_{p}-C_{p}\left(\mathrm{FeGa}_{3}\right)$ is presented in the inset of Fig. 8(a) in a standard $\Delta C_{p} / T(T)$ representation. It shows a broad feature with a maximum at about $5 \mathrm{~K}$ that resembles a two-level Schottky anomaly. A reasonable description of the experimental data is achieved assuming that the anomaly in $\Delta C_{p} / T$ originates from thermally activated transitions between two discrete levels having the same degeneracy, separated by an energy gap $\Delta / k_{\mathrm{B}} \approx 16 \mathrm{~K}$ [see the inset of Fig. 8(a)]. An additional small constant term of $2 \mathrm{~mJ} \mathrm{~mol}^{-1} \mathrm{~K}^{-2}$ has also been included to improve the quality of the fit. Although this small contribution to the $C_{p}$ superimposed on the Schottky anomaly mimics a Fermi-liquid-like specific 
heat, the insulating temperature dependence of the resistivity overrules the presence of a finite density of states at the Fermi level in $\mathrm{Fe}_{0.92} \mathrm{Mn}_{0.08} \mathrm{Ga}_{3}$. Therefore, we suppose that this additional specific heat overlapping with the gap anomaly has a more complex temperature dependence and originates from low-energy magnetic excitations and/or localized in-gap states.

Similar Schottky-type anomalies in the specific heat are visible for all the compounds in the $\mathrm{Fe}_{1-x} \mathrm{Mn}_{x} \mathrm{Ga}_{3}$ series [see Fig. 8(b)] as well as for $\mathrm{FeGa}_{3-y} \mathrm{Zn}_{y}$ (not shown). They occur at the same temperature for both dopants, regardless of the doping level $x$. In order to estimate the entropy associated with this excess specific heat in the doped crystals, we calculated $\Delta C_{p}(T)$ for each crystal by subtracting from the total $C_{p}(T)$ the lattice contribution approximated by the specific heat of $\mathrm{FeGa}_{3}$. We extrapolated $\Delta C_{p} / T$ to $T=0$ and then integrated $\Delta C_{p}(T) / T$ up to $T^{*}=15 \mathrm{~K}$, at which temperature the specific heat of the doped crystals becomes very similar to that of $\mathrm{FeGa}_{3}$ :

$$
S_{\mathrm{m}}=\int_{0}^{T^{*}} \frac{\Delta C_{p}}{T} d T .
$$

The values of the entropy $S_{\mathrm{m}}$ recovered at $15 \mathrm{~K}$ increase nearly linearly with increasing doping level, starting from $x \approx 1.5 \%$, and follow the same straight line for both Mn- and $\mathrm{Zn}$-doped $\mathrm{FeGa}_{3}$, as indicated in the inset of Fig. 8(c). The slope of this line corresponds to an entropy of about $0.4 R \ln 2$ per $\mathrm{Mn} / \mathrm{Zn}$ atom introduced by the doping.

Although this entropy is strongly dominated by transitions between two states, it contains also additional contributions originating presumably from low-energy magnetic excitations and localized in-gap states in the vicinity of the Fermi level. The insulating behavior of the resistivity with a VRH conduction at low temperatures in $\mathrm{Mn}$ - and $\mathrm{Zn}$-doped $\mathrm{FeGa}_{3}$ excludes the presence of delocalized holes in the whole investigated doping ranges. The broad features in $\Delta C_{p} / T$ with maxima at the temperature of $0.7 \mathrm{~K}$ grow with doping in both $\mathrm{FeGa}_{3-y} \mathrm{Zn}_{y}$ and $\mathrm{Fe}_{1-x} \mathrm{Mn}_{x} \mathrm{Ga}_{3}$, which may be suggestive of an approaching quantum critical point.

Doping of $\mathrm{FeGa}_{3}$ with $\mathrm{Mn}$ and $\mathrm{Zn}$ has also very similar effects on the overall magnetic properties. For both dopants, the magnetic susceptibility in the temperature range of $\sim 100$ $400 \mathrm{~K}$ can be described by a modified Curie law

$$
\chi(T)=\chi_{0}+\frac{C}{T},
$$

where $\chi_{0}$ denotes the temperature-independent part of the magnetic susceptibility and $C$ is the Curie constant defined as $C=N_{\mathrm{m}} p_{\mathrm{eff}}^{2} /\left(3 k_{\mathrm{B}}\right)$ with $N_{\mathrm{m}}$ being the number of magnetic atoms per formula unit and $p_{\text {eff }}$ denoting their effective magnetic moment. The least-squares fits to the experimental data [see Figs. 9(a) and 9(b)] yield values of $\chi_{0}$ ranging from $-5 \times 10^{-5} \mathrm{emu} / \mathrm{mol}$ to $-2 \times 10^{-5} \mathrm{emu} / \mathrm{mol}$, which are similar to the diamagnetic signals observed for crystals of undoped $\mathrm{FeGa}_{3}$. Values of the effective magnetic moment per formula unit $p_{\text {eff }}$ derived from the Curie constant are plotted as a function of the measured doping level in Fig. 9(c).

For both $\mathrm{Fe}_{1-x} \mathrm{Mn}_{x} \mathrm{Ga}_{3}$ and $\mathrm{FeGa}_{3-y} \mathrm{Zn}_{y}$, the effective moment increases gradually with increasing doping level. The effective moment calculated per dopant is close to the theoretical value of $1.73 \mu_{\mathrm{B}} /$ dopant expected for $\operatorname{spin} S=\frac{1}{2}$ [see Fig. 9(d)]. Inclusion of a paramagnetic Weiss temperature $\theta$ did not lead to any significant improvement of the fits. Thus, the magnetic susceptibility measurements indicate that doping with both $\mathrm{Zn}$ and $\mathrm{Mn}$ introduces moments of $S=\frac{1}{2}$ per dopant atom, which are freely fluctuating at temperatures above $\sim 100 \mathrm{~K}$. The magnetic anisotropy in this temperature range is reflected only by smaller absolute values of $\chi_{0}$ for $H$ applied in the $a b$ plane than in case of $H \| c$, imitating the magnetic anisotropy found for the undoped $\mathrm{FeGa}_{3}$.

At lower temperatures, the magnetic susceptibilities of the doped crystals deviate from the Curie-Weiss law in a manner that may indicate an increasing role for antiferromagnetic interactions. To follow the variation of the effective moments with temperature, we plot the temperature dependence of the product $\left(\chi-\chi_{0}\right) T$ measured in a magnetic field of 70 kOe [insets, Figs. 9(a) and 9(b)]. For $\mathrm{Fe}_{1-x} \mathrm{Mn}_{x} \mathrm{Ga}_{3}$ and $\mathrm{FeGa}_{3-y} \mathrm{Zn}_{y}$, at temperatures above $\sim 80 \mathrm{~K}$ the product $\left(\chi-\chi_{0}\right) T$ is nearly temperature independent, defining the Curie law regime. At lower temperatures, $\left(\chi-\chi_{0}\right) T$ decreases strongly with decreasing temperature, implying a decrease in the effective fluctuating moment.

Below $\sim 30 \mathrm{~K}$, the magnetic susceptibilities of both $\mathrm{Fe}_{1-x} \mathrm{Mn}_{x} \mathrm{Ga}_{3}$ and $\mathrm{FeGa}_{3-y} \mathrm{Zn}_{y}$ develop substantial magnetic anisotropies and become field dependent in magnetic fields $H$ applied along the $c$ axis (See Fig. S2 in the Supplemental Material [64]). Figure 10(a) presents the low-temperature magnetic susceptibility data for $\mathrm{Fe}_{0.92} \mathrm{Mn}_{0.08} \mathrm{Ga}_{3}$. The $\chi(T)$ measured in weak magnetic fields applied along the $c$ axis continues to increase with decreasing temperature. Its temperature dependence at $T \lesssim 5 \mathrm{~K}$ can be approximated by $\chi(T) \sim T^{-\alpha}$ with $\alpha \approx 0.5$, as indicated in the inset of Fig. 10(a). Application of $H \gtrsim 1$ kOe leads to a gradual suppression of the upturn in $\chi(T)$ at temperatures below $\sim 10$ $\mathrm{K}$. In contrast, the magnetic susceptibility for $H \|[110]$ is almost field independent from 1 to $70 \mathrm{kOe}$ even at lowest temperatures. It shows a broad and very weak maximum at $T \approx 5 \mathrm{~K}$

To check for the presence of a spontaneous magnetization, we measured the magnetization as a function of magnetic field at constant temperatures [Fig. 10(b)]. In the inset of Fig. 10(b), we replotted the isothermal $M(H)$ curves obtained in fields applied along the $c$ axis as $M^{2}$ against $H / M$. The absence of a positive $M^{2}$ intercept in this Arrott plot indicates that there is no spontaneous magnetization in $\mathrm{Fe}_{0.92} \mathrm{Mn}_{0.08} \mathrm{Ga}_{3}$. Importantly, the $M(H)$ curves at $T=1.8 \mathrm{~K}$ do not saturate in magnetic fields up to $70 \mathrm{kOe}$, as expected for a system with noninteracting moments. They increase nearly linearly with increasing field strength in the high-field range, independent of the field direction, and the maximum measured moment for $\mathrm{Fe}_{0.92} \mathrm{Mn}_{0.08} \mathrm{Ga}_{3}$ in the field of $70 \mathrm{kOe}$ is only $\sim 0.028 \mu_{\mathrm{B}} /$ f.u. and $\sim 0.023 \mu_{\mathrm{B}} /$ f.u. for $H \|[001]$ and $H \|[110]$, respectively. Implications of these field dependencies of the magnetization will be discussed in Sec. IV.

Similar results were obtained for crystals of $\mathrm{Fe}_{1-x} \mathrm{Mn}_{x} \mathrm{Ga}_{3}$ and $\mathrm{FeGa}_{3-y} \mathrm{Zn}_{y}$ regardless of the dopant type and level, with nonsaturating $M(H)$ at $T=1.8 \mathrm{~K}, \chi(T)$ showing broad maxima at $T \lesssim 5 \mathrm{~K}$ for $H \|[110]$ and $\chi(T) \sim T^{-\alpha}$ at $T \lesssim 5 \mathrm{~K}$ in the case of an applied field along the $c$ axis with $\alpha$ between 0.2 and 0.6 . Although the exact values of the exponent $\alpha$ may be affected by slight crystal misalignment and the presence of 

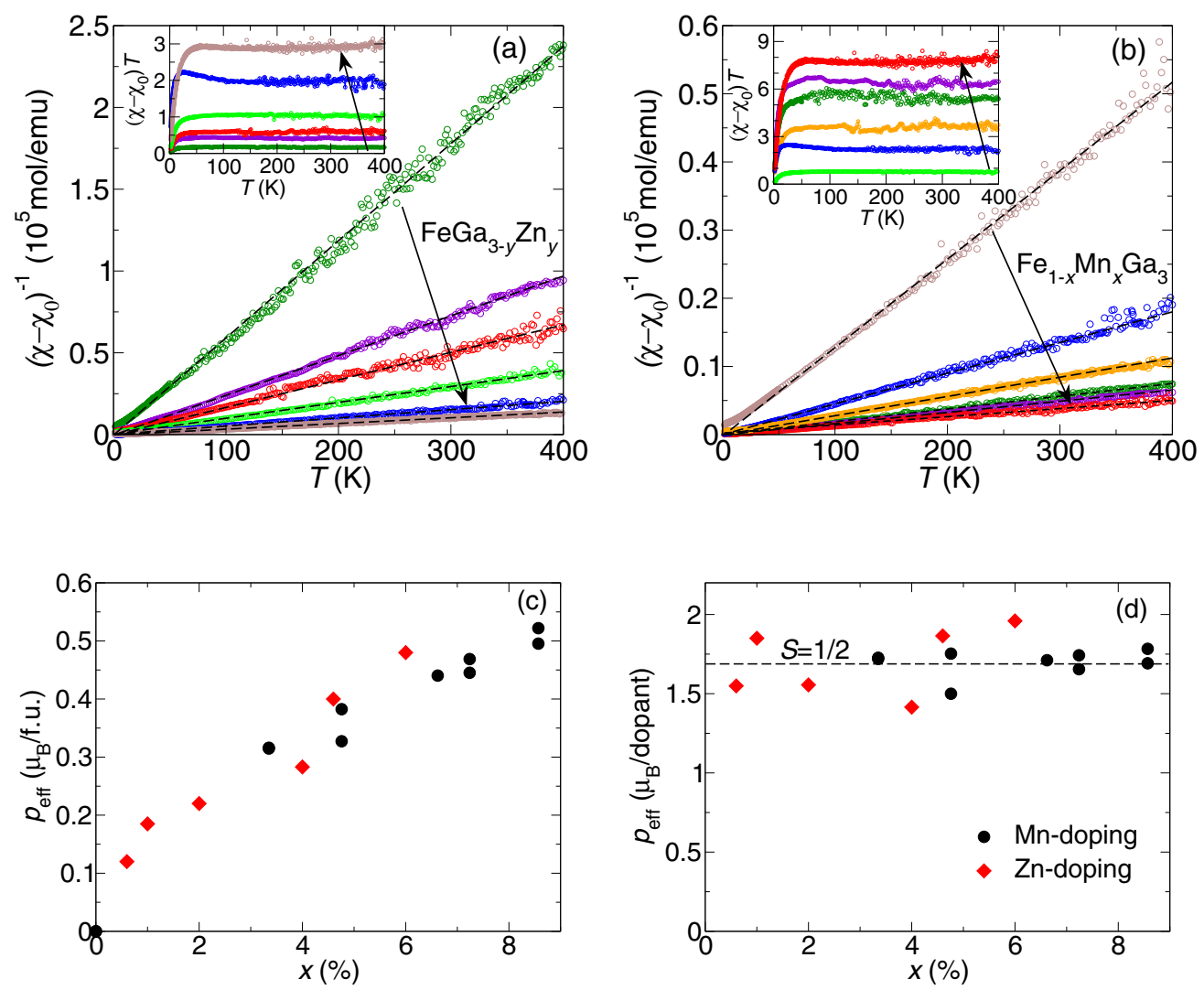

FIG. 9. (Color online) Inverse magnetic susceptibilities for $\mathrm{FeGa}_{3-y} \mathrm{Zn}_{y}(y=0.006 ; 0.01 ; 0.02 ; 0.04 ; 0.046 ; 0.06)(\mathrm{a})$ and $\mathrm{Fe}_{1-x} \mathrm{Mn}_{x} \mathrm{Ga}_{3}$ $(x=0.032 ; 0.043 ; 0.044 ; 0.061 ; 0.065 ; 0.066 ; 0.085 ; 0.087)(b)$ after subtracting a constant term $\chi_{0}$ as obtained from fits using a modified Curie law (see details in text). The insets in panels (a) and (b) show the values of the product $\chi T$ as functions of temperature for the field of $H=70 \mathrm{kOe}$ obtained after subtracting the temperature-independent terms $\chi_{0}$. Arrows indicate the direction of the increase in measured doping level. Panel (c) depicts the values of the effective magnetic moment per formula unit obtained from the fits as a function of the doping level $x$ for $\mathrm{FeGa}_{3-y} \mathrm{Zn}_{y}$ (red diamonds) and $\mathrm{Fe}_{1-x} \mathrm{Mn}_{x} \mathrm{Ga}_{3}$ (black circles). The values of the effective moment recalculated per dopant are shown in panel (d), together with a dashed line indicating an effective moment of $1.73 \mu_{\mathrm{B}}$, as expected for $S=\frac{1}{2}$.

a small amount of paramagnetic impurities, they are always below 1 . Such weak power laws in $\chi(T)$ at $T \lesssim 5 \mathrm{~K}$ may hint at the emergence of critical fluctuations associated with a proximity to a quantum critical point in the presence of disorder introduced by doping [65]. Further, no hysteresis was observed in field-cooled (FC) and zero-field-cooled (ZFC) $\chi(T)$ data for $\mathrm{Fe}_{1-x} \mathrm{Mn}_{x} \mathrm{Ga}_{3}$ and $\mathrm{FeGa}_{3-y} \mathrm{Zn}_{y}$, that could indicate a spin-glass behavior at low temperatures. The values of the magnetic susceptibility, measured at a temperature of $2 \mathrm{~K}$, increase systematically with increasing doping level
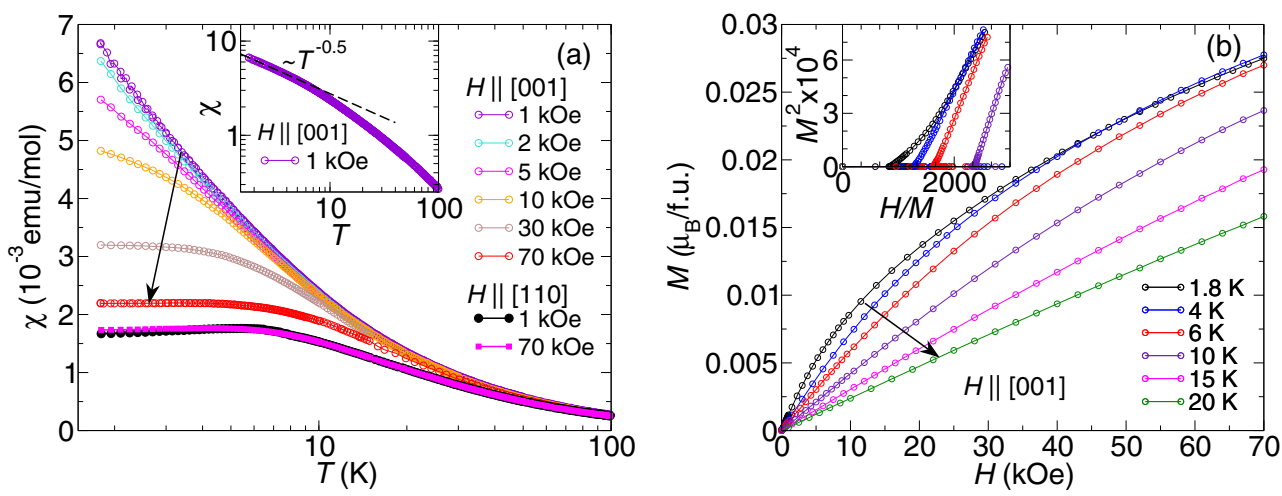

FIG. 10. (Color online) (a) Magnetic susceptibility of $\mathrm{Fe}_{0.92} \mathrm{Mn}_{0.08} \mathrm{Ga}_{3}$ measured in various magnetic fields applied either along the $c$ axis (empty circles) or along the [110] direction (full squares). Arrows point in the direction of the increasing magnetic field applied along the $c$ axis. For $H \|[110], \chi(T)$ is only weakly field dependent. The inset shows $\chi(T)$ measured in $H=1 \mathrm{kOe}$ applied along the $c$ axis in a log-log plot. The dashed line represents the fit to the data at $T \lesssim 5 \mathrm{~K}$ using $\chi(T) \sim T^{-\alpha}$. (b) Magnetization measured for $\mathrm{Fe}_{0.92} \mathrm{Mn}_{0.08} \mathrm{Ga}_{3}$ in a magnetic field applied along the $c$ axis at indicated temperatures. The inset shows the $M(H)$ curves plotted in a standard Arrott representation. 


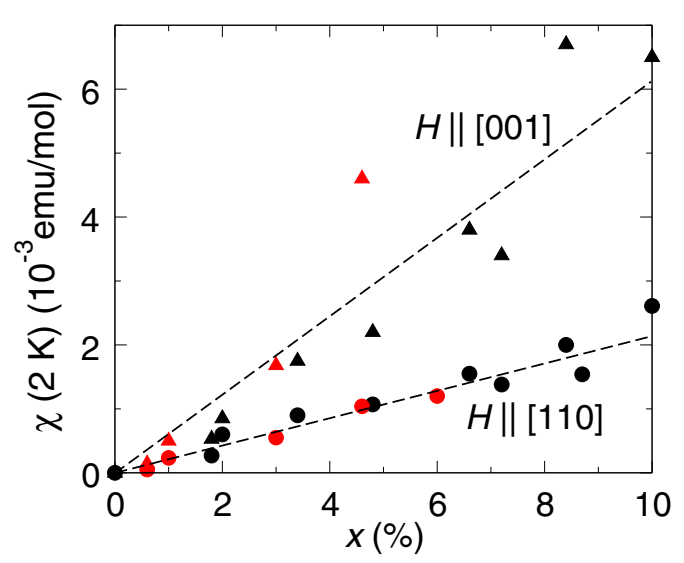

FIG. 11. (Color online) Values of the magnetic susceptibility for $\mathrm{Fe}_{1-x} \mathrm{Mn}_{x} \mathrm{Ga}_{3}$ (black) and $\mathrm{FeGa}_{3-y} \mathrm{Zn}_{y}$ (red) measured at $2 \mathrm{~K}$ in a magnetic field applied either along the [110] direction (circles) or along the $c$ axis for $H=1 \mathrm{kOe}$ (triangles). Dashed lines are guides for the eye.

for both investigated directions of magnetic field, following the same straight lines for crystals of $\mathrm{Mn}$ - and $\mathrm{Zn}$-doped $\mathrm{FeGa}_{3}$ (Fig. 11). These results need to be contrasted with previous studies on polycrystals of $\mathrm{Fe}_{1-x} \mathrm{Mn}_{x} \mathrm{Ga}_{3}$ which revealed that already in samples with $x=0.05$ there are either two magnetic phase transitions at temperatures of $\sim 10$ and $\sim 30 \mathrm{~K}$ according to Ref. [66] or there is an onset of ferromagnetism with $T_{\mathrm{C}} \approx 160 \mathrm{~K}$ superimposed on a CurieWeiss-type paramagnetic behavior with the effective magnetic moment of $4.9 \mu_{\mathrm{B}} / \mathrm{Mn}$ [67].

Finally, neutron powder diffraction experiments have been performed to inspect the magnetic and structural properties of hole-doped $\mathrm{FeGa}_{3}$ at low temperatures. The diffraction patterns for $\mathrm{Fe}_{0.95} \mathrm{Mn}_{0.05} \mathrm{Ga}_{3}$ measured at temperatures of 1.5 and $15 \mathrm{~K}$ are basically identical (Fig. 12). There are no differences in the sizes and positions of either magnetic or nuclear peaks that could indicate a change in the long-range magnetic order or a structural transition in the doped $\mathrm{FeGa}_{3}$ at low temperatures. Furthermore, the magnetic peaks observed

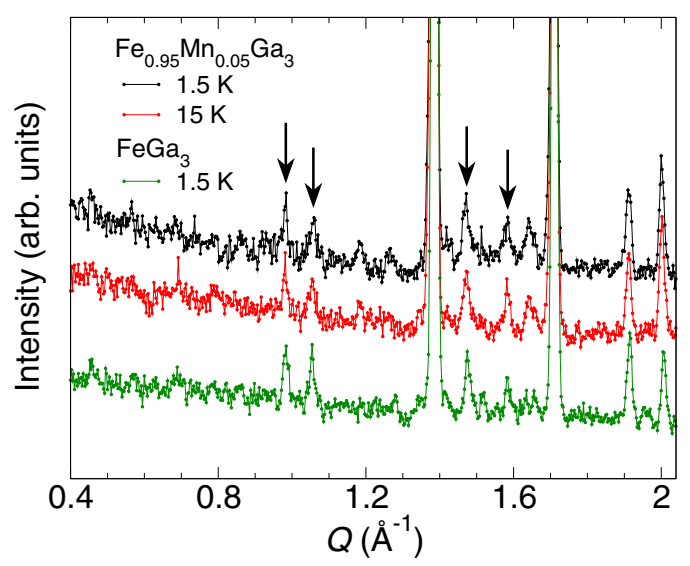

FIG. 12. (Color online) Neutron powder diffraction patterns for $\mathrm{Fe}_{0.95} \mathrm{Mn}_{0.05} \mathrm{Ga}_{3}$, obtained at temperatures of $1.5 \mathrm{~K}$ (black) and $15 \mathrm{~K}$ (red), together with the data for undoped $\mathrm{FeGa}_{3}$ collected at $1.5 \mathrm{~K}$. The main magnetic peaks are indicated by arrows.

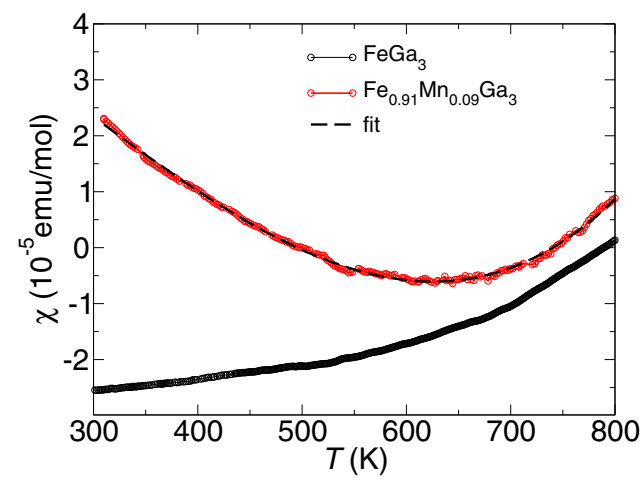

FIG. 13. (Color online) Magnetic susceptibility of $\mathrm{Fe}_{0.91} \mathrm{Mn}_{0.09} \mathrm{Ga}_{3}$ (red circles) measured at temperatures above $\sim 300 \mathrm{~K}$ in a magnetic field of $10 \mathrm{kOe}$ on a set of randomly oriented crystals, together with the data for $\mathrm{FeGa}_{3}$ (black circles) calculated as explained in the text. Dashed line represents the fit to a model described in the text.

in the neutron powder diffraction patterns for $\mathrm{Fe}_{0.95} \mathrm{Mn}_{0.05} \mathrm{Ga}_{3}$ persist at temperatures up to $300 \mathrm{~K}$ and, most importantly, are similar to those found for undoped $\mathrm{FeGa}_{3}$ (Fig. 12), indicating that their magnetic structures are closely related.

A magnetization study carried out at high temperatures shows that for hole-doped $\mathrm{FeGa}_{3}$, the fluctuating moment behavior observed at $T \gtrsim 100 \mathrm{~K}$ gives way to a notable increase in $\chi(T)$ at temperatures above $\sim 600 \mathrm{~K}$. Figure 13 presents the temperature dependence of the magnetic susceptibility for $\mathrm{Fe}_{0.91} \mathrm{Mn}_{0.09} \mathrm{Ga}_{3}$, measured on a set of randomly oriented single crystals in a magnetic field of $10 \mathrm{kOe}$, together with the inferred polycrystalline magnetic susceptibility for undoped $\mathrm{FeGa}_{3}$ estimated as $\chi(T)=\frac{1}{3}\left[2 \chi_{a b}(T)+\chi_{c}(T)\right]$, where $\chi_{a b}(T)$ and $\chi_{c}(T)$ denote the magnetic susceptibilities measured in magnetic fields applied in the $a b$ plane and along the $c$ axis, respectively. The $\chi(T)$ for $\mathrm{Fe}_{0.91} \mathrm{Mn}_{0.09} \mathrm{Ga}_{3}$ can be well described by an activation-type behavior, extended by an additional term $C / T$ that is introduced to account for the presence of fluctuating moments (Fig. 13). The value of the Curie constant $C$ derived from this fit is very similar to that obtained from the analysis of the magnetic susceptibility at temperatures between 100 and $300 \mathrm{~K}$, and $\chi_{0}=-5.4 \times 10^{-5} \mathrm{emu} \mathrm{mol}^{-1}$. Most importantly, the obtained spin gap $\Delta_{S}=0.31 \mathrm{eV}$ is only slightly smaller than that estimated for the parent compound $\mathrm{FeGa}_{3}$, implying that the doping with $\mathrm{Mn}$ in $\mathrm{Fe}_{1-x} \mathrm{Mn}_{x} \mathrm{Ga}_{3}$ falls short of closing the spin gap.

\section{DISCUSSION}

There is an experimental consensus that $\mathrm{FeGa}_{3}$ is an insulator with a narrow gap of $0.4 \mathrm{eV}[31,32,41]$. The band gap can be well reproduced in first-principles electronicstructure calculations based on DFT in its standard LDA and GGA implementations [33,40,43]. These calculations, however, result in a nonmagnetic state, whereas our neutron diffraction measurements revealed a complex type of magnetic order in $\mathrm{FeGa}_{3}$, even at room temperature. The existence of staggered moments ultimately questions the LDA description 

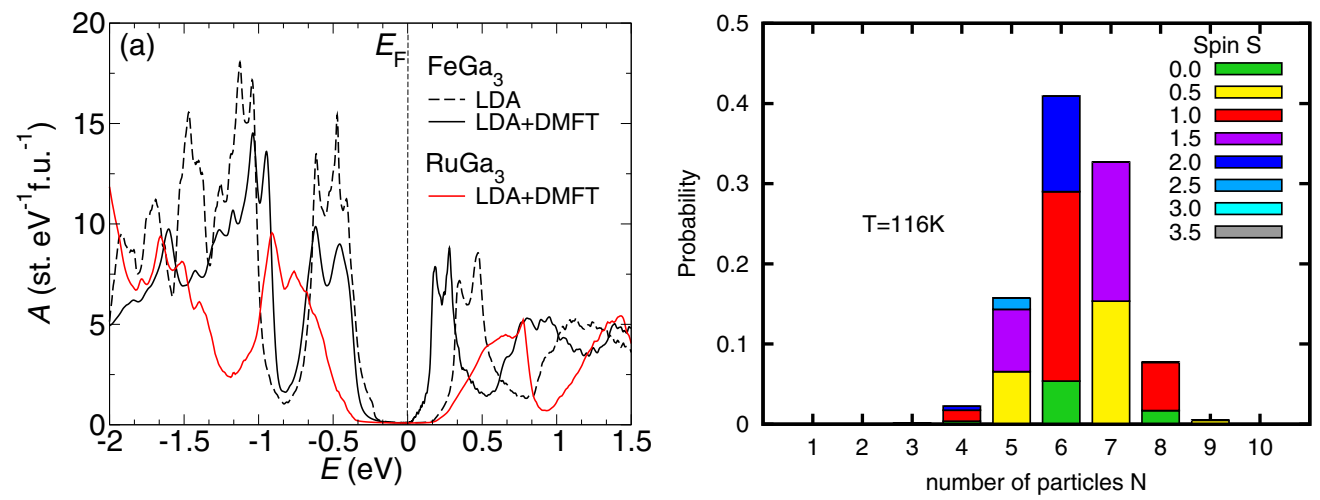

FIG. 14. (Color online) Left panel shows LDA+DMFT spectral functions calculated at $T=116 \mathrm{~K}$ (solid lines) for FeGa 3 (black) and for $\mathrm{RuGa}_{3}$ (red) compared to the LDA results (dashed line). Right panel presents probability of atomic states of the DMFT impurity at $T=116 \mathrm{~K}$, decomposed in number of particles $N$ ( $x$ axis) and spin state $S$, as indicated.

of $\mathrm{FeGa}_{3}$ as a nonmagnetic band insulator and calls for more sophisticated computational techniques.

Both the LDA calculations and the ARPES study show a narrow Fe $3 d$-derived state located near the top of the valence band $[31,33,41]$, indicating the relevance of electronic correlation effects. Following this observation, Yin and Pickett [33] applied a static mean-field-like LSDA $+U$ method and found a magnetic insulating ground state for $\mathrm{FeGa}_{3}$, independent of which double-counting scheme was used, with an antiferromagnetic order being lowest in energy [33]. Further, the size of the band gap obtained assuming moderate values of the onsite Coulomb interaction for the Fe $3 d$ states, $U \sim 2 \mathrm{eV}$, coincides with experimental results [33]. However, a nonmagnetic state is stabilized for $U \lesssim 1.5 \mathrm{eV}$ and even for larger values of $U$ if screening effects are included in the LSDA $+U$ formalism via a Yukawa ansatz [36]. These findings indicate that the $3 d$ electrons in $\mathrm{FeGa}_{3}$ are not close to the well-understood limits of being either localized or itinerant. Consequently, neither LDA nor $\mathrm{L}(\mathrm{S}) \mathrm{DA}+U$ methods are able to provide a satisfying description of its electronic band structure. Therefore, we employed DMFT to interpolate between the strongly localized and delocalized limits pertinent to our analysis of $\mathrm{FeGa}_{3}$. We applied the combination of the DFT in its LDA approximation with DMFT to investigate the charge and spin states of the iron atoms as well as temperaturedependent many-body renormalizations in $\mathrm{FeGa}_{3}$. In order to separate effects originating from correlated $3 d$ states of iron, we performed calculations also for the isostructural and isoelectronic compound $\mathrm{RuGa}_{3}$.

The left panel of Fig. 14 shows the LDA+DMFT spectral functions for both $\mathrm{FeGa}_{3}$ and $\mathrm{RuGa}_{3}$, together with the bandtheory (LDA) results. Overall, our calculations reproduce insulating states for $\mathrm{FeGa}_{3}$ and $\mathrm{RuGa}_{3}$ with a narrow gap produced by a strong hybridization between $d$ states of $\mathrm{Fe}$ or $\mathrm{Ru}$ and $p$ states of $\mathrm{Ga}$. The size of the band gap obtained from band theory for $\mathrm{FeGa}_{3}$ of $0.35 \mathrm{eV}$ is close to results of the combined PES and inverse PES study [31], and also previous works $[31,41,43]$. Further, the shape of the DOSs calculated within the LDA approximation for both $\mathrm{FeGa}_{3}$ and $\mathrm{RuGa}_{3}$ is in a very good agreement with antecedent reports $[31,33,41,43,68]$.

Inclusion of many-body effects via LDA+DMFT leads to a slight decrease in the size of the charge gap in $\mathrm{FeGa}_{3}$, as compared to the LDA results [Fig. 14(a)]. To further inspect the strength of dynamical correlations, we calculate the effective masses from the energy derivative of the DMFT self-energy at the Fermi level. The formal evaluation based on the first linear regime in the DMFT self-energy shows that the effective masses are rather low and nearly temperature independent $m^{\text {DMFT }} / m^{\text {LDA }} \approx 1.3-1.4$ (see Fig. 15 ). This indicates a large degree of delocalization of the states at the bottom of the valence band and only weak dynamical correlations.

To address the charge and spin states of $\mathrm{Fe}$ in $\mathrm{FeGa}_{3}$, we analyze the probability distribution of the many-body wave function with respect to the eigenstates of the effective iron atom, decomposed into the number of particles $N$ and the spin state $S$. The histogram of the CTQMC (the right panel of Fig. 14) shows the largest probability for the spin state $S=1$, with a variance $\delta S=\left\langle(S-\langle S\rangle)^{2}\right\rangle \approx 0.34$ that reflects strong fluctuations at short time scales. The dominance of the $S=1$ configuration implies that $\mathrm{FeGa}_{3}$ is not a nonmagnetic insulator with $\mathrm{Fe}^{2+}$ in a low-spin state, as previously suggested [32,34-37,39-43]. Further, the number of $\mathrm{Fe} 3 d$ electrons $N_{\mathrm{d}} \approx 6.3$ has a large variance $\delta N=\left\langle(N-\langle N\rangle)^{2}\right\rangle \approx 0.88$, implying a strong mixed valent character. Although the variances in $S$ and $N$ are large, they

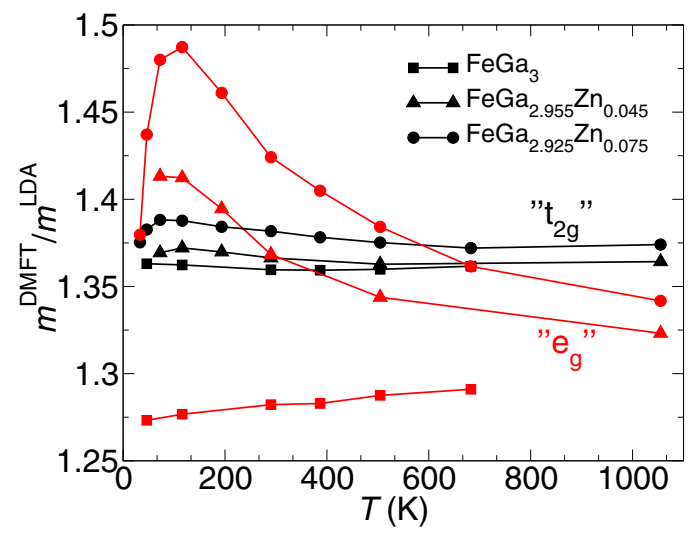

FIG. 15. (Color online) LDA+DMFT effective masses for doped and undoped $\mathrm{FeGa}_{3}$ as obtained from the energy derivative of the selfenergy. Orbital components that account for the majority of spectral weight for the valence (conduction) states are denoted " $t_{2 \mathrm{~g}}$ " in black (" $e_{\mathrm{g}}$ " in red). 

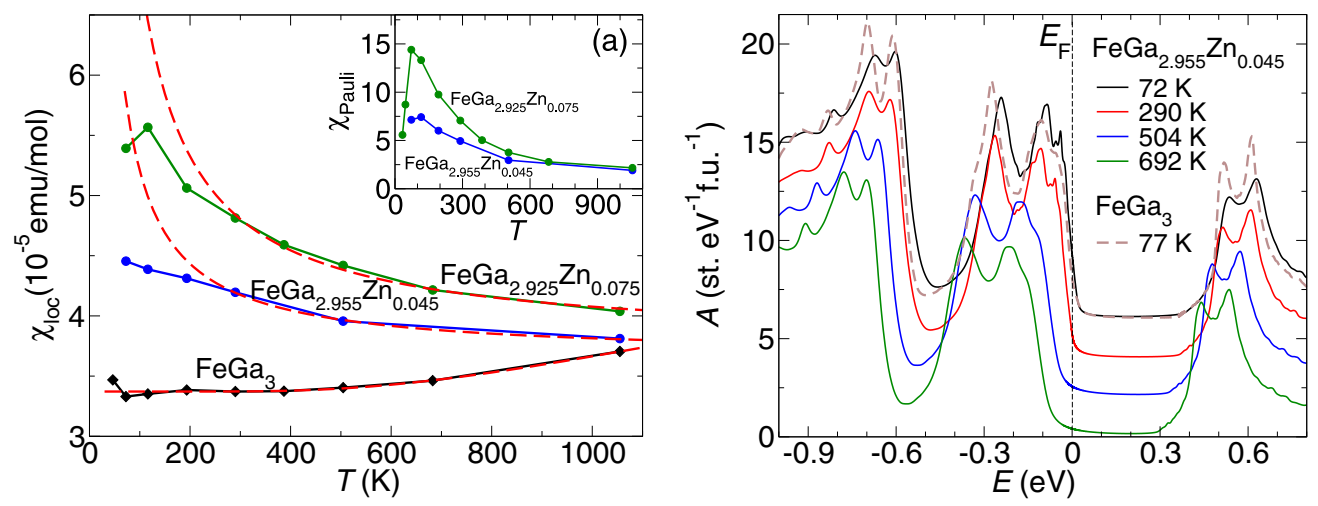

FIG. 16. (Color online) (a) LDA+DMFT local spin susceptibility as functions of temperature (solid lines) for FeGa $\mathrm{Fa}_{2.95} \mathrm{Zn}_{0.045}$ (blue), $\mathrm{FeGa}_{2.925} \mathrm{Zn}_{0.075}$ (green), and $\mathrm{FeGa}_{3}$ (black) together with fits (dashed lines) to either the modified Curie law or to the activation law $\chi(T)=$ $\chi_{0}+\exp \left[-\Delta_{\mathrm{S}} /\left(k_{\mathrm{B}} T\right)\right]$ with a spin gap $\Delta_{\mathrm{S}}=0.21 \mathrm{eV}$. The inset displays Pauli susceptibilities computed from the spectral function for the two simulated $\mathrm{Zn}$-doping levels in the same units. (b) LDA+DMFT spectral functions (solid lines) for FeGa $\mathrm{Ga}_{2.925} \mathrm{Zn}_{0.075}$ at different temperatures compared to those for $\mathrm{FeGa}_{3}$ at $77 \mathrm{~K}$ (dashed line). An offset (multiples of $2 \mathrm{st}^{-\mathrm{eV}^{-1} \text { f.u. }}{ }^{-1}$ ) was added to each curve so that all the presented curves can be easily viewed and compared. The $A(E)$ curve for $\mathrm{FeGa}_{3}$ was shifted on the energy scale by $-0.365 \mathrm{eV}$ to facilitate visual comparison of its shape with the calculated $A(E)$ for $\mathrm{FeGa}_{2.955} \mathrm{Zn}_{0.045}$.

do not evolve with temperature. Thus, the charge and spin states of $\mathrm{Fe}$ are temperature independent in $\mathrm{FeGa}_{3}$.

The experimental magnetic susceptibility of $\mathrm{FeGa}_{3}$ is only weakly temperature dependent below $\sim 500 \mathrm{~K}$ and increases strongly at higher temperatures, indicative of a gap in the spin excitation spectrum (Fig. 3). Early attempts to explain this behavior were based on thermal excitations of electrons across the band gap [39]. These models, however, require unreasonably large values of the density of states near the band-gap edges to account for the size of the experimental $\chi(T)$ [39]. This, together with the absence of similar spin excitations in the magnetic susceptibility of $\mathrm{RuGa}_{3}$ [an insulator with a slightly larger band gap of $\sim 0.5 \mathrm{eV}$ (Fig. 14)], makes these density of states models implausible. In turn, the lack of temperature dependence of the spin state of $\mathrm{Fe}$ in $\mathrm{FeGa}_{3}$, together with magnetic order revealed by our neutron diffraction measurements, overrules the so-called localized moment model proposed by Tsujii et al. [39], in which thermally induced transitions from the nonmagnetic ground state to the first excited state with $S=1$ determine the shape of $\chi(T)$ for $\mathrm{FeGa}_{3}$ at high temperatures.

To get insight into the energy scales for spin excitations in $\mathrm{FeGa}_{3}$ based on our LDA+DMFT study, we calculated local spin susceptibilities for both $\mathrm{FeGa}_{3}$ and $\mathrm{RuGa}_{3}, \chi_{\mathrm{loc}}(\omega=0) \sim$ $\int d \tau\left\langle S_{z}(\tau) S_{z}(0)\right\rangle$. For $\mathrm{FeGa}_{3}$, the $\chi_{\mathrm{loc}}(T)$ can be approximated by the sum of a small constant term of $3.4 \times 10^{-5} \mathrm{emu} / \mathrm{mol}$ and a contribution with an activation-type temperature dependence due to a gap of $0.21 \mathrm{eV}$ [Fig. 16(a)]. This spin gap results from quenching of spin fluctuations at finite time scales due to the formation of a coherent insulating state at low temperatures [10]. It is smaller than the spin gap of $0.33-0.41 \mathrm{eV}$ that was estimated from the activation expression applied to the experimental $\chi(T)$. The larger size of the experimental spin gap as compared to that obtained from the local spin susceptibility suggests the relevance of nonlocal exchange interactions that are not treated in our single-site DMFT setup. We suppose that these intersite exchange interactions are also responsible for the antiferromagnetic order revealed by our neutron diffraction measurements since the LDA+DMFT calculations do not give any hint for this antiferromagnetism in $\mathrm{FeGa}_{3}$. Importantly, the temperature dependence of the local susceptibility, derived from the LDA+DMFT study, is more than one order of magnitude smaller than the experimental values for the uniform susceptibility (Fig. 3). Hence, the temperature-induced fluctuating moment of the underlying spin state is presumably not local [43], and a considerable momentum dependence in $\chi$ is expected. A similar disparity in size between the measured uniform magnetic susceptibility and the calculated $\chi_{\mathrm{loc}}(T)$ was recently reported for $\mathrm{FeSi}$, an archetypal correlated band insulator [10]. Neutron experiments for $\mathrm{FeSi}$ found a significant magnetic scattering at ferromagnetic reciprocal lattice vectors [69], confirming the nonlocal charater of the temperature-induced magnetic moment. In contrast, for $\mathrm{RuGa}_{3}$ the local susceptibility is very small $\left(\sim 1.15 \times 10^{-5} \mathrm{emu} / \mathrm{mol}\right)$, as expected for its more delocalized $4 d$ electrons, and it increases only very weakly with increasing temperature, in line with the experimental results (see Fig. 3).

Although our neutron powder diffraction study for $\mathrm{FeGa}_{3}$ revealed a complex magnetic ordering with an onset above room temperature, there is no signature of a magnetic phase transition in $\chi(T)$ measured up to $900 \mathrm{~K}$. For numerous low-dimensional spin systems, however, features in $\chi(T)$ associated with magnetic ordering are not pronounced, especially in case of magnetic transitions at high temperatures. The shape of the temperature dependence of the magnetic susceptibility of such systems is determined mostly by the strongest exchange interaction. Consequently, signatures of magnetic transitions in $\chi(T)$ can be only barely detected even for compounds with staggered moments as large as $2-3 \mu_{\mathrm{B}}$ per magnetic ion [70,71]. For $\mathrm{FeGa}_{3}$ the expected ordered moments are much smaller, the ordering temperature is above $300 \mathrm{~K}$, and the temperature dependence of the magnetic susceptibility is determined by the presence of the large gap in the spin excitation spectrum. Further, LSDA $+U$ calculations suggest that there is a very strong antiferromagnetic coupling 
$(J \approx 3000 \mathrm{~K}$ ) between Fe atoms within structural dimers, that leads to the formation of spin singlets [33]. Nevertheless, we can not exclude that the Néel temperature for $\mathrm{FeGa}_{3}$ is higher than $900 \mathrm{~K}$ and therefore no signature of the magnetic ordering was found in $\chi(T)$ in the investigated temperature range.

Given the rather small staggered moments and an incommensurate ordering wave vector, we considered the possibility that the additional peaks in neutron diffraction patterns originate from extrinsic sources, such as the artifacts of the measurement process or a small amount of an impurity phase. The former can be excluded because neutron diffraction measurements performed in two different cryostats gave the same results. Furthermore, the diffraction patterns collected for samples of $\mathrm{FeGa}_{3}$ and $\mathrm{Fe}_{0.95} \mathrm{Mn}_{0.05} \mathrm{Ga}_{3}$ show very similar peaks at small values of the wave vector $Q$. Following, we discuss the possibility of secondary phases as extrinsic sources of these additional diffraction peaks.

Both the neutron and x-ray powder diffraction patterns collected on the same sample of $\mathrm{FeGa}_{3}$ did not show any unexpected peaks or extra intensities that could be assigned to the nuclear structure of an impurity phase. We found only a few small reflections that can be unambiguously assigned to the presence of $\sim 2 \mathrm{vol} . \%$ of $\alpha-\mathrm{Ga}$, which is nonmagnetic. Moreover, magnetization measurements performed on several random crystals gave only a weakly temperature-dependent diamagnetic signal, further ruling out the presence of magnetic impurity phases, including iron oxides. Such measurements are much more sensitive to magnetic impurities than the neutron powder diffraction experiments. Microstructures of several crystals were also studied using a scanning electron microscope with resolutions down to $\sim 10 \mathrm{~nm}$. Examples of high-resolution images of as-grown crystalline surfaces taken in the backscattered electrons mode are presented in Fig. S1 in the Supplemental Material [64]. They show no trace of a secondary phase or compositional inhomogeneities, apart from small and single inclusions of gallium flux. Since backscattered electrons are sensitive to local variations in the atomic number, the presence of an impurity phase would have been visible as either brighter or lighter fields in the surface images. Finally, the sample of $\mathrm{FeGa}_{3}$ was prepared only from crystals grown in batches with nominal ratio $\mathrm{Fe}: \mathrm{Ga}$ $=1: 12.5$. As expected based on the Fe-Ga phase diagram [48], the only binary phase found in these batches was $\mathrm{FeGa}_{3}$. The next binary phase $\mathrm{Fe}_{3} \mathrm{Ga}_{4}$ was observed only in growths with nominally less than 85 at. \% Ga. The presence of this compound in the sample investigated by neutron diffraction was ruled out based on $\mathrm{X}$-ray powder diffraction, where the detection limit is of the order of 1 vol.\%. Furthermore, magnetic peaks expected for $\mathrm{Fe}_{3} \mathrm{Ga}_{4}$ [72] do not match the extra peaks observed in the neutron patterns of $\mathrm{FeGa}_{3}$. Taken together, our results argue strongly against extrinsic sources of the additional peaks in the neutron powder diffraction patterns of $\mathrm{FeGa}_{3}$ and $\mathrm{Fe}_{0.95} \mathrm{Mn}_{0.05} \mathrm{Ga}_{3}$.

We note that room-temperature ${ }^{57} \mathrm{Fe}$ Mössbauer spectra for $\mathrm{FeGa}_{3}$ did not show a distinct magnetic splitting, thus ruling out the presence of a sizable hyperfine field on Fe site. For an incommensurate system with small ordered moments, however, the presence of a finite internal magnetic field may manifest itself only in a broadening of spectral lines due to a distribution of hyperfine fields arising from the incommensurately modulated magnetic structure [73]. This effect superposed on a quadrupole splitting $\Delta E=0.33 \mathrm{~mm} / \mathrm{s}$ in $\mathrm{FeGa}_{3}[39,45]$ may be too weak to be detected on top of broadening originating from lifetime effects and experimental resolution.

The antiferromagnetic order in $\mathrm{FeGa}_{3}$ with the ordering wave vector incommensurate with the nuclear structure and only small staggered moments suggests a close similarity of $\mathrm{FeGa}_{3}$ to Slater insulators. In the case of Slater insulators, however, an opening of a band gap is induced by the formation of a spin-density wave, whereas our computational study shows that the magnetic order is not needed to cause the insulating behavior in $\mathrm{FeGa}_{3}$. Moreover, electronic-structure calculations based on local density methods give a similar gap of $0.4 \mathrm{eV}$ for both a nonmagnetic and a magnetically ordered state in $\mathrm{FeGa}_{3}$ [33], thus making a spin-density-wave-type order resulting from a Fermi surface instability in $\mathrm{FeGa}_{3}$ highly unlikely. Therefore, we conclude that the modulated magnetic structure in $\mathrm{FeGa}_{3}$ results rather from competing exchange interactions.

It is of interest to compare $\mathrm{FeGa}_{3}$ with the prototypal correlated band insulator FeSi. Both compounds are band insulators with hybridization gaps of $0.4 \mathrm{eV}$ for $\mathrm{FeGa}_{3}$ and $0.05 \mathrm{eV}$ for FeSi [10,17,31]. Electronic-structure calculations based on LDA+DMFT indicate a strong mixed valence of $\mathrm{Fe}$ with a similar average occupancy for the Fe $3 d$ shell of 6.3 and 6.2 in $\mathrm{FeGa}_{3}$ and $\mathrm{FeSi}$ [10], respectively. Furthermore, the spin state with $S=1$ dominates in both $\mathrm{FeGa}_{3}$ and $\mathrm{FeSi}$, with strong fluctuations on short-time scales. Even the remarkably uniform distribution of states is very similar in the two compounds (see Fig. 14 and Fig. S5 of Ref. [10]). For both systems, the spin excitations are gapped, and the temperature-induced fluctuating moment is, to a large extent, not local. The large degree of delocalization and strong momentum dependence is typical for correlated band insulators and differentiates them from $f$-electron-based Kondo insulators such as $\mathrm{Ce}_{3} \mathrm{Bi}_{4} \mathrm{Pt}_{3}$, $\mathrm{SmB}_{6}, \mathrm{YbB}_{12}$, or CeNiSn [20]. Also, the ratio between the size of the band gap $E_{g}$ and the width of the bands around the gap $E_{g} / W \gg 1$ for $\mathrm{FeGa}_{3}$ and $\mathrm{FeSi}$, whereas for Kondo insulators $E_{g} / W \ll 1$ [20]. Further, values of the $U / W$ ratio estimated for both $\mathrm{FeGa}_{3}$ and $\mathrm{FeSi}$ are of the order of 1, much smaller than those found in strongly correlated electron systems [10]. The latter require strong electron-electron interactions to open a band gap at the Fermi level. The effective mass renormalization resulting from many-body effects in $\mathrm{FeGa}_{3}$ is rather low, $m^{\mathrm{DMFT}} / m^{\mathrm{LDA}} \approx 1.3-1.4$, but it is comparable to that in FeSi $m^{\mathrm{DMFT}} / m^{\mathrm{LDA}} \approx 1.5[8,10,17]$. For $\mathrm{FeGa}_{3}$, however, the effective masses are nearly independent of temperature (Fig. 15). This has to be contrasted with FeSi, for which the same local interaction vertex gives rise to a temperature dependence in the self-energy that is pertinent for experimental observables [10]. Consequently, calculations based on DMFT indicate that FeSi metallizes with increasing temperature through correlation-induced incoherence [10]. The primary difference between $\mathrm{FeGa}_{3}$ and FeSi lies in the size of the band gap, which is about eight times larger for $\mathrm{FeGa}_{3}$ than for FeSi. Therefore, for FeSi all the relevant energy scales are of similar magnitude resulting in low-energy properties being dominated by correlation effects [10], whereas for $\mathrm{FeGa}_{3}$ the effects of local physics are rather small on a relative energy 
scale. Furthermore, in $\mathrm{FeGa}_{3}$, nonlocal exchange interactions are presumably responsible for the antiferromagnetic order revealed by our neutron diffraction study. In contrast, for FeSi the influence of nonlocal exchange is reflected mostly in an increase in size of the band gap [74]. Neutron diffraction measurements did not give any sign of a spin ordering in $\mathrm{FeSi}$ even at lowest temperatures [75].

We were successful in incorporating sizable amounts of $\mathrm{Zn}$ and $\mathrm{Mn}$ into $\mathrm{FeGa}_{3}$. This p-type doping on both $\mathrm{Fe}$ and Ga sites did not lead either to a notable change in the long-range magnetic order or to a metal-insulator transition. Remarkably, the effect of doping is very similar for both $\mathrm{Mn}$ and $\mathrm{Zn}$. Electrical resistivity measurements show that the doping creates localized states in the semiconducting gap. Our magnetization study indicates that both a substitution of $\mathrm{Zn}$ onto the $\mathrm{Ga}$ site and a replacement of Fe by $\mathrm{Mn}$ lead to formation of an essentially noninteracting magnetic moment of $S=\frac{1}{2}$ that fluctuates freely at temperatures above $\sim 80 \mathrm{~K}$ (Fig. 9). At lower temperatures, the effective moment decreases strongly with decreasing temperature, regardless of the dopant type and level.

Similar effects of doping were observed for simple semiconductors such as silicon and germanium. The thermodynamic properties of these systems in the insulating regime can be well described by the phenomenological model introduced by Bhatt and Lee [76]. The Bhatt-Lee model [77] considers local moments of $S=\frac{1}{2}$ associated with dopant atoms, which lead to the Curie-type behavior in $\chi(T)$ at high temperatures and a weaker temperature dependence of the magnetic susceptibility at lower temperatures due to antiferromagnetic exchange interactions between these randomly distributed spins. Bhatt and Lee iteratively divided the system into a hierarchy of antiferromagnetically coupled spin pairs and showed that at low temperatures $\chi(T) \propto T^{-\alpha}$ and $C_{p}(T) \propto$ $T^{1-\alpha}$ with $\alpha<1$ [77]. For $\mathrm{Fe}_{1-x} \mathrm{Mn}_{x} \mathrm{Ga}_{3}$ and $\mathrm{FeGa}_{3-y} \mathrm{Zn}_{y}$, however, at temperatures below $\sim 30 \mathrm{~K}$, the magnetic susceptibilities develop notable magnetic anisotropies. Additionally, the magnetic susceptibilities measured in $H \| a b$ are nearly field independent and saturate at $T \lesssim 6 \mathrm{~K}$. Furthermore, estimates of the magnetic specific heat from the experimental $\chi(T)$ based on the Bhatt-Lee model [Eqs. (2) and (3) of Ref. [77]] give values that are at least about one order of magnitude smaller than the experimentally obtained magnetic specific heat even at the lowest temperatures (not shown). Finally, there is an important difference between $\mathrm{FeGa}_{3}$ and the simple semiconductors regarding doping levels required to drive the system through an insulator-to-metal transition: For $\mathrm{Si}$ doped with phosphorus (boron), the critical concentration of dopant atoms $N_{\mathrm{c}}=3.52 \times 10^{18} \mathrm{~cm}^{-3}\left(4.06 \times 10^{18} \mathrm{~cm}^{-3}\right)$ corresponds to about 0.007 at. \% (0.009 at. \%), whereas even the introduction of about 2.5 at. $\%$ of acceptors in $\mathrm{Fe}_{0.9} \mathrm{Mn}_{0.1} \mathrm{Ga}_{3}$ does not lead to a metallic state. Also, for the archetypal correlated band insulators, FeSi and $\mathrm{FeSb}_{2}$, similar or smaller concentrations of dopants were found to induce a metallic conduction in $\mathrm{FeSi}_{1-x} \mathrm{Al}_{x}, \mathrm{Fe}_{1-x} \mathrm{Co}_{x} \mathrm{Si}$, $\mathrm{Fe}_{1-x} \mathrm{Mn}_{x} \mathrm{Si}, \mathrm{Fe}_{1-x} \mathrm{Co}_{x} \mathrm{Sb}_{2}, \mathrm{FeSb}_{2-x} \mathrm{Sn}_{x}, \mathrm{Fe}_{1-x} \mathrm{Ni}_{x} \mathrm{Sb}_{2}$, and $\mathrm{FeSb}_{2-x} \mathrm{Te}_{x}[20,21,23,24,78-82]$.

The development of a broad Schottky-type anomaly in specific heat of both $\mathrm{Fe}_{1-x} \mathrm{Mn}_{x} \mathrm{Ga}_{3}$ and $\mathrm{FeGa}_{3-y} \mathrm{Zn}_{y}$ indicates the formation of a gap (Fig. 8). The magnitude of this gap
$\Delta / k_{\mathrm{B}} \approx 16 \mathrm{~K}$ is very similar for $\mathrm{Mn}$ - and $\mathrm{Zn}$-doped $\mathrm{FeGa}_{3}$ and does not change appreciably with increasing doping level. Since the gap anomalies in specific heat are accompanied by the broad features in magnetic susceptibility, we considered their common origin as due to thermally induced changes in spin state of dopant atoms from $S=0$ to $\frac{1}{2}$. Attempts to describe the $\chi(T)$ based on this model, however, do not lead to satisfactory fits (not shown). Instead, the magnetic susceptibilities revealed notable magnetic anisotropies in this temperature range, very similar for both $\mathrm{Fe}_{1-x} \mathrm{Mn}_{x} \mathrm{Ga}_{3}$ and $\mathrm{FeGa}_{3-y} \mathrm{Zn}_{y}$. We propose that holes introduced to $\mathrm{FeGa}_{3}$ by doping with $\mathrm{Mn}$ and $\mathrm{Zn}$ localize at low temperatures into magnetic "droplets" on the scale of the lattice spacing due to strong exchange interactions between spins of these carriers and spins of $\mathrm{Fe}$ in the antiferromagnetic lattice. Such localized charge carriers accompanied by reorientations of surrounding lattice spins are usually referred to as small spin polarons. Thus, we interpret the gap found in specific-heat measurements as the binding energy of these states forming at low temperatures.

The linear increase of the excess entropy associated with the Schottky anomalies with increasing doping levels observed for $\mathrm{Mn}$ - and $\mathrm{Zn}$-doped $\mathrm{FeGa}_{3}$ [Fig. 8(c)] is consistent with a growing number of doping-induced polarons. Also, the magnetic susceptibilities at the lowest temperatures increase nearly linearly with the dopant levels for both $\mathrm{Fe}_{1-x} \mathrm{Mn}_{x} \mathrm{Ga}_{3}$ and $\mathrm{FeGa}_{3-y} \mathrm{Zn}_{y}$ (Fig. 11), in line with the scenario of magnetic polarons. Further, the field-dependent magnetization $M(H)$ curves measured at $1.85 \mathrm{~K}$ do not saturate up to the highest field of $70 \mathrm{kOe}$, and the maximum measured moments correspond to only $\sim 0.3 \mu_{\mathrm{B}}$ per dopant atom [Fig. 10(b)]. Since the doping with $\mathrm{Mn}$ and $\mathrm{Zn}$ falls short of closing the spin gap of $0.4 \mathrm{eV}$ and essentially does not influence the antiferromagnetic ordering as revealed by our neutron diffraction measurements, the shape of the $M(H)$ curves reflects the magnetization associated with the dopant atoms. The lack of saturation and only small values of the field-induced magnetic moment per dopant atom that are independent of the doping level suggest the presence of strong exchange interactions between spins of carriers introduced by the doping and spins of $\mathrm{Fe}$ atoms in the antiferromagnetic material. This exchange coupling is presumably responsible for the formation of the spin polarons. Recent muon spin rotation study gave an evidence for the presence of anisotropic spin polarons in $\mathrm{FeGa}_{3}$ at temperatures below $\sim 10 \mathrm{~K}$ [44]. However, we caution that spectroscopic measurements on $\mathrm{Mn}$ - and $\mathrm{Zn}$-doped $\mathrm{FeGa}_{3}$ are needed to place our proposal on firmer footing.

Our experimental study for $\mathrm{Mn}$ - and $\mathrm{Zn}$-doped $\mathrm{FeGa}_{3}$ is in strong contradiction with predictions based on electronicstructure calculations within DFT, which indicated the formation of an itinerant ferromagnetic state with half-metallic properties in hole-doped $\mathrm{FeGa}_{3}$ over an extended composition range, independent of the presence of preformed Fe moments in the undoped semiconducting phase [43]. To test this theoretical prediction and to further study the influence of hole doping on magnetic properties and many-body renormalizations, we employed DMFT. We simulated doping of $\mathrm{FeGa}_{3}$ with holes on both Fe and Ga sites in LDA+DMFT calculations using the VCA approximation.

Our calculations indicate that hole doping on either $\mathrm{Fe}$ or $\mathrm{Ga}$ sites has very similar effects on the electronic 
structure and magnetic properties of $\mathrm{FeGa}_{3}$. Figure 16(a) shows $\chi_{\text {loc }}(T)$ calculated for a few selected doping levels. At high temperatures, the local susceptibilities can be well described using a modified Curie law. With decreasing temperature, a notable deviation from $\chi_{\text {loc }}(T) \sim T^{-1}$ develops. The values of $\chi_{\text {loc }}(T)$ become smaller than expected from the Curie law, capturing the behavior observed in the experiments. For both $\mathrm{Fe}_{1-x} \mathrm{Mn}_{x} \mathrm{Ga}_{3}$ and $\mathrm{FeGa}_{3-y} \mathrm{Zn}_{y}$, however, modified Curie fits to $\chi_{\mathrm{loc}}(T)$ at temperatures above $\sim 300 \mathrm{~K}$ result in small values of the effective moment per dopant, much smaller than the moment of $1.73 \mu_{\mathrm{B}}$ expected for $S=\frac{1}{2}$. For example, for $\mathrm{FeGa}_{2.925} \mathrm{Zn}_{0.075}$, we obtained only $p_{\text {eff }}=0.55 \pm 0.02 \mu_{\mathrm{B}}$ per Zn.

As seen in Fig. 15, the deviation of $\chi_{\operatorname{loc}}(T)$ from the modified Curie law is accompanied by a notable increase in the effective mass for the orbital character that accounts for the majority of spectral weight at the Fermi level, near the top of the valence band. Although the overall values of $m^{\text {DMFT }} / m^{\text {LDA }}$ for the simulated doping levels are not very big, they are comparable with those found for FeSi [10]. Most importantly, the temperature-induced changes are as large as $15 \%$ an increase in the shown range for $\mathrm{FeGa}_{2.925} \mathrm{Zn}_{0.075}$. Conceptually, growing effective masses can be expected to increase the validity of a local picture of fluctuating spin moments. Therefore, the experimentally observed decrease of the effective moment when lowering temperature points towards a predominantly itinerant mechanism for the spin response at all temperatures.

Moreover, the changes in $\chi_{\text {loc }}$ coincide with the temperature dependence of the spectral weight at the Fermi level displayed in Fig. 16(b). The Pauli susceptibility [see the inset in Fig. 16(a)] calculated from

$$
\chi_{\text {Pauli }}(T)=\mu_{\mathrm{B}}^{2} N_{\mathrm{A}} \int d E\left(-\frac{\partial f(E)}{\partial E}\right) A(E)
$$

provides an even better match to the experimental uniform susceptibility than the LDA+DMFT local spin susceptibility. Here, $N_{\mathrm{A}}$ is Avogadro's number, $\mu_{\mathrm{B}}$ is the Bohr magneton, $f(E)$ denotes the Fermi function, $A(\mathrm{E})$ is the spectral function. These calculations imply that the physics of local moments is not predominant in the $\mathrm{Mn}$ - and $\mathrm{Zn}$-doped $\mathrm{FeGa}_{3}$.

To further address the strength of magnetic correlations in the hole-doped $\mathrm{FeGa}_{3}$ based on DMFT, we calculate also the Sommerfeld-Wilson ratio

$$
R_{\mathrm{SW}}=\frac{4 \pi^{2} k_{\mathrm{B}}^{2}}{3\left(g \mu_{\mathrm{B}}\right)^{2}} \frac{\chi_{\mathrm{p}}}{\gamma} .
$$

Here, $g=2$ is the electron Lande factor, $\gamma$ is the Sommerfeld coefficient obtained based on the calculated spectrum at the Fermi level and $\chi_{p}$ is approximated by the local spin susceptibility $\chi_{\text {loc }}$. A tentative $T \rightarrow 0$ extrapolation gives values of the $R_{\mathrm{SW}}$ in the range of $1-3$ for both $\mathrm{FeGa}_{2.955} \mathrm{Zn}_{0.045}$ and $\mathrm{FeGa}_{2.925} \mathrm{Zn}_{0.075}$, thus indicating the absence of strong electronic spin-spin interactions which could lead to Stonertype magnetic instability, in line with our experimental results.

Our experimental and computational study puts forward the complex magnetic properties of the $\mathrm{Fe}_{1-x} \mathrm{Mn}_{x} \mathrm{Ga}_{3}$ and $\mathrm{FeGa}_{3-y} \mathrm{Zn}_{y}$ solid solutions. Even though the inclusion of dopant atoms basically does not influence the long-range antiferromagnetic order and falls short of closing the spin (and charge) gap, it triggers an intriguing magnetic behavior. Both the experimental $\chi(T)$ and the calculated $\chi_{\text {loc }}$ exhibit a Curie-type behavior at high temperatures and become smaller than expected based on the Curie law at lower temperatures. However, the experimental magnetic susceptibility suggests the formation of local moments of $S=\frac{1}{2}$ associated with the dopant atoms that fluctuate freely at temperatures above $\sim 100 \mathrm{~K}$ (Fig. 9), whereas the LDA+DMFT calculations point to the itinerant character of the spin response, that however mimic a Curie-type fluctuating moment behavior at high temperatures. These findings, together with the presence of an incommensurate magnetic order with rather small staggered moments as well as the formation of anisotropic spin polaroniclike states at low temperatures, indicate that $\mathrm{Fe}_{1-x} \mathrm{Mn}_{x} \mathrm{Ga}_{3}$ and $\mathrm{FeGa}_{3-y} \mathrm{Zn}_{y}$ combine features of both itinerant and localized magnetism.

We notice that our LDA+DMFT calculations as well as a previous computational study based on DFT [43] indicate that $p$-type doping shifts the Fermi level to the valence band, whereas transport measurements for all $\mathrm{Fe}_{1-x} \mathrm{Mn}_{x} \mathrm{Ga}_{3}$ and $\mathrm{FeGa}_{3-y} \mathrm{Zn}_{y}$ revealed only insulating behavior with electrical conductivities determined by the presence of localized in-gap states even for the highest doping levels. It is known, however, that computational methods based on the local density and the VCA approximation can not address this type of conduction in doped semiconductors $[83,84]$. In addition, intersite exchange interactions between spins of carriers introduced by the doping and spins of magnetic ions need to be accounted for in case of the formation of small magnetic polarons. Inclusion of these interactions in the DMFT calculations would at least necessitate a cluster extension [85].

\section{CONCLUSIONS}

Our neutron powder diffraction, thermodynamic, and electrical resistivity measurements show that $\mathrm{FeGa}_{3}$ is an insulator in which iron moments order above room temperature in a complex antiferromagnetic structure that is incommensurate with the nuclear lattice and spin excitations are gapped. Based on many-body calculations within the framework of DMFT, we claim that, while the iron atoms in $\mathrm{FeGa}_{3}$ are dominantly in an $S=1$ state, there are strong and temperature-independent charge and spin fluctuations at short-time scales, indicating a strongly delocalized character of $3 d$ electrons. Further, the low magnitude of local contributions to the spin susceptibility advocates a predominantly itinerant mechanism for the spin response. Our combined experimental and computational investigations indicate that $\mathrm{FeGa}_{3}$ is a correlated band insulator with only small effects of many-body renormalizations, in which nonlocal exchange interactions are presumably responsible for the spin gap of $0.4 \mathrm{eV}$ and the antiferromagnetic order.

Calculations based on DMFT indicate that dynamical correlation effects in $\mathrm{FeGa}_{3}$ become stronger as a result of hole doping. Using electrical resistivity and thermodynamic measurements as well as neutron powder diffraction, we establish that doping of $\mathrm{FeGa}_{3}$ with $\mathrm{Mn}$ and $\mathrm{Zn}$ neither affects the long-range antiferromagnetic ordering nor leads to an insulator-to-metal transition. The effect of doping is very similar for both $\mathrm{Mn}$ and $\mathrm{Zn}$. The temperature dependence 
of the electrical resistivity indicates that localized states are formed in the semiconducting gap. Magnetization study shows that the introduction of each hole is accompanied by the formation of a spin moment of $S=\frac{1}{2}$, that fluctuates freely at temperatures above $\sim 100 \mathrm{~K}$ and decreases gradually with lowering temperature. While these experimental findings suggest the existence of local moments associated with the dopant atoms, the LDA+DMFT calculations argue for an itinerant character of spin excitations that, however, mimic a Curie-type behavior of the $\chi(T)$ at high temperatures. At low temperatures, it is tempting to interpret the thermodynamic and transport properties of the $\mathrm{Mn}$ - and $\mathrm{Zn}$ doped $\mathrm{FeGa}_{3}$ in terms of small spin polarons formed by holes that localize into magnetic "droplets" due to strong exchange couplings between spins of these free carriers and Fe moments in the antiferromagnetic lattice. Consequently, our study indicates that $\mathrm{Fe}_{1-x} \mathrm{Mn}_{x} \mathrm{Ga}_{3}$ and $\mathrm{FeGa}_{3-y} \mathrm{Zn}_{y}$ combine features of both itinerant and localized magnetism due to a complex interplay between nonlocal exchange and correlation effects.

\section{ACKNOWLEDGMENTS}

Work at Brookhaven National Laboratory (M.G., A.P., and M.C.A.) was carried out under the auspices of the US Department of Energy, Office of Basic Energy Sciences, under Contract No. DE-AC02-98CH1886. Research at Rutgers University (J.M.T. and G.K.) was sponsored by the Department of Defense National Security Science and Engineering Faculty Fellowship via the Air Force Office of Scientific Research. J.M.T. and G.K. were further supported by the NSF Grant No. DMR 1308141.
[1] V. Jaccarino, G. K. Wertheim, J. H. Wernick, L. R. Walker, and S. Arajs, Phys. Rev. 160, 476 (1967).

[2] C. Petrovic, J. W. Kim, S. L. Budko, A. I. Goldman, P. C. Canfield, W. Choe, and G. J. Miller, Phys. Rev. B 67, 155205 (2003); A. Perucchi, L. Degiorgi, R. Hu, C. Petrovic, and V. F. Mitrovic, Eur. Phys. J. B 54, 175 (2006).

[3] R. Hu, R. P. Hermann, F. Grandjean, Y. Lee, J. B. Warren, V. F. Mitrovic, and C. Petrovic, Phys. Rev. B 76, 224422 (2007).

[4] V. I. Anisimov, S. Y. Ezhov, I. S. Elfimov, I. V. Solovyev, and T. M. Rice, Phys. Rev. Lett. 76, 1735 (1996).

[5] A. V. Lukoyanov, V. V. Mazurenko, V. I. Anisimov, M. Sigrist, and T. M. Rice, Eur. Phys J. B. 53, 205 (2006).

[6] Y. Takahashi and T. Moriya, J. Phys. Soc. Jpn. 46, 1451 (1979).

[7] O. Delaire, K. Marty, M. B. Stone, P. R. C. Kent, M. S. Lucas, D. L. Abernathy, D. Mandrus, and B. C. Sales, Proc. Natl. Acad. Sci. USA 108, 4725 (2011).

[8] V. V. Mazurenko, A. O. Shorikov, A. V. Lukoyanov, K. Kharlov, E. Gorelov, A. I. Lichtenstein, and V. I. Anisimov, Phys. Rev. B 81, 125131 (2010).

[9] V. I. Anisimov, R. Hlubina, M. A. Korotin, V. V. Mazurenko, T. M. Rice, A. O. Shorikov, and M. Sigrist, Phys. Rev. Lett. 89, 257203 (2002).

[10] J. M. Tomczak, K. Haule, and G. Kotliar, Proc. Natl. Acad. Sci. USA 109, 3243 (2012).

[11] J. M. Tomczak, K. Haule, and G. Kotliar, in New Materials for Thermoelectric Applications: Theory and Experiment, NATO Science for Peace and Security Series B: Physics and Biophysics, edited by V. Zlatic and A. Hewson (Springer, Netherlands, 2013), p. 45.

[12] J. M. Tomczak, K. Haule, T. Miyake, A. Georges, and G. Kotliar, Phys. Rev. B 82, 085104 (2010).

[13] C. Fu and S. Doniach, Phys. Rev. B 51, 17439 (1995).

[14] M. Sentef, J. Kunes, P. Werner, and A. P. Kampf, Phys. Rev. B 80, 155116 (2009).

[15] J. Kuneš and V. I. Anisimov, Phys. Rev. B 78, 033109 (2008).

[16] P. Sun, N. Oeschler, S. Johnsen, B. B. Iversen, and F. Steglich, Phys. Rev. B 79, 153308 (2009).

[17] J. Beille, J. Voiron, and M. Roth, Solid State Commun. 47, 399 (1983); D. Mandrus, J. L. Sarrao, A. Migliori, J. D. Thompson, and Z. Fisk, Phys. Rev. B 51, 4763 (1995); S. Paschen, E. Felder, M. A. Chernikov, L. Degiorgi, H. Schwer, H. R. Ott, D. P. Young, J. L. Sarrao, and Z. Fisk, ibid. 56, 12916 (1997); T. Saso and K. Urasaki, J. Phys. Chem. Solids 63, 1475 (2002); M. Arita, K. Shimada, Y. Takeda, M. Nakatake, H. Namatame, M. Taniguchi, H. Negishi, T. Oguchi, T. Saitoh, A. Fujimori, and T. Kanomata, Phys. Rev. B 77, 205117 (2008); H. Yamaoka, M. Matsunami, R. Eguchi, Y. Ishida, N. Tsujii, Y. Takahashi, Y. Senba, H. Ohashi, and S. Shin, ibid. 78, 045125 (2008); D. Plencner and R. Hlubina, ibid. 79, 115106 (2009).

[18] Z. Schlesinger, Z. Fisk, H.-T. Zhang, and M. B. Maple, Phys. B (Amsterdam) 237-238, 460 (1997).

[19] S. Yeo, S. Nakatsuji, A. D. Bianchi, P. Schlottmann, Z. Fisk, L. Balicas, P. A. Stampe, and R. J. Kennedy, Phys. Rev. Lett. 91, 046401 (2003).

[20] M. K. Chattopadhyay, S. B. Roy, and S. Chaudhary, Phys. Rev. B 65, 132409 (2002); M. A. Chernikov, L. Degiorgi, E. Felder, S. Paschen, A. D. Bianchi, H. R. Ott, J. L. Sarrao, Z. Fisk, and D. Mandrus, ibid. 56, 1366 (1997).

[21] J. F. DiTusa, K. Friemelt, E. Bucher, G. Aeppli, and A. P. Ramirez, Phys. Rev. B 58, 10288 (1998).

[22] C. Petrovic, Y. Lee, T. Vogt, N. Dj. Lazarov, S. L. Budko, and P. C. Canfield, Phys. Rev. B 72, 045103 (2005).

[23] A. Bentien, G. K. H. Madsen, S. Johnsen, and B. B. Iversen, Phys. Rev. B 74, 205105 (2006).

[24] R. Hu, V. F. Mitrovic, and C. Petrovic, Phys. Rev. B 74, 195130 (2006).

[25] R. Hu, V. F. Mitrovic, and C. Petrovic, Phys. Rev. B 76, 115105 (2007).

[26] A. Bentien, S. Johnsen, G. K. H. Madsen, B. B. Iversen, and F. Steglich, Europhys. Lett. 80, 17008 (2007).

[27] P. Sun, N. Oeschler, S. Johnsen, Bo B. Iversenb, and F. Steglich, Dalton Trans. 39, 1012 (2010).

[28] A. K. L. Fan, G. H. Rosenthal, H. L. McKinzie, and A. Wold, J. Solid State Chem. 5, 136 (1972).

[29] Y. Nishino, M. Kato, S. Asano, K. Soda, M. Hayasaki, and U. Mizutani, Phys. Rev. Lett. 79, 1909 (1997).

[30] A. Ślebarski, M. B. Maple, E. J. Freeman, C. Sirvent, D. Tworuszka, M. Orzechowska, A. Wrona, A. Jezierski, 
S. Chiuzbaian, and M. A. Neumann, Phys. Rev. B 62, 3296 (2000); A. Ślebarski, J. Phys. D: Appl. Phys. 39, 856 (2006).

[31] M. Arita, K. Shimada, Y. Utsumi, O. Morimoto, H. Sato, H. Namatame, M. Taniguchi, Y. Hadano, and T. Takabatake, Phys. Rev. B 83, 245116 (2011).

[32] Y. Hadano, S. Narazu, M. A. Avila, T. Onimaru, and T. Takabatake, J. Phys. Soc. Jpn. 78, 013702 (2009).

[33] Z. P. Yin and W. E. Pickett, Phys. Rev. B 82, 155202 (2010).

[34] E. M. Bittar, C. Capan, G. Seyfarth, P. G. Pagliuso, and Z. Fisk, J. Phys.: Conf. Ser. 200, 012014 (2010).

[35] K. Umeo, Y. Hadano, S. Narazu, T. Onimaru, M. A. Avila, and T. Takabatake, Phys. Rev. B 86, 144421 (2012).

[36] J. M. Osorio-Guillen, Y. D. Larrauri-Pizarro, and G. M. Dalpian, Phys. Rev. B 86, 235202 (2012).

[37] N. Haldolaarachchige, A. B. Karki, W. Adam Phelan, Y. M. Xiong, R. Jin, Julia Y. Chan, S. Stadler, and D. P. Young, J. Appl. Phys. 109, 103712 (2011).

[38] T. Takabatake, F. Iga, T. Yoshino, Y. Echizen, K. Katoh, K. Kobayashi, M. Higa, N. Shimizu, Y. Bando, G. Nakamoto, H. Fujii, K. Izawa, T. Suzuki, T. Fujita, M. Sera, M. Hiroi, K. Maezawa, S. Mock, H. v. Löhneysen, A. Brückl, K. Neumaier, and K. Andres, J. Magn. Magn. Mater. 177-181, 277 (1998).

[39] N. Tsujii, H. Yamaoka, M. Matsunami, R. Eguchi, Y. Ishida, Y. Senba, H. Ohashi, S. Shin, T. Furubayashi, H. Abe, and H. Kitazawa, J. Phys. Soc. Jpn. 77, 024705 (2008).

[40] Y. Imai and A. Watanabe, Intermetallics 14, 722 (2006).

[41] U. Häussermann, M. Boström, P. Viklund, Ä. Rapp, and T. Björnängen, J. Solid State Chem. 165, 94 (2002).

[42] Y. Amagai, A. Yamamoto, T. Iida, and Y. Takanashi, J. Appl. Phys. 96, 5644 (2004).

[43] D. J. Singh, Phys. Rev. B 88, 064422 (2013).

[44] V. G. Storchak, J. H. Brewer, R. L. Lichti, R. Hu, and C. Petrovic, J. Phys.: Condens. Matter. 24, 185601 (2012).

[45] G. L. Whittle, P. E. Clark, and R. Cywinski, J. Phys. F: Met. Phys. 10, 2093 (1980).

[46] A. A. Gippius, V. Yu. Verchenko, A. V. Tkachev, N. E. Gervits, C. S. Lue, A. A. Tsirlin, N. Büttgen, W. Krätschmer, M. Baenitz, M. Shatruk, and A. V. Shevelkov, Phys. Rev. B 89, 104426 (2014).

[47] V. Petricek, M. Dusek, and L. Palatinus, Jana, The Crystallographic Computing System (Institute of Physics, Praha, Czech Republic, 2006).

[48] C. Dasarathy and W. Hume-Rothery, Proc. R. Soc. London, Ser. A 286, 141 (1965).

[49] M. Wagner-Reetz, R. Cardoso-Gil, and Yu. Grin, J. Electron. Mater, doi:10.1007/s11664-013-2888-1 (2013).

[50] J. Rodriguez-Carvajal, Phys. B (Amsterdam) 192, 55 (1993).

[51] P. Blaha, K. Schwarz, G. Madsen, D. Kvasnicka, and J. Luitz, WIEN2K, an Augmented Plane Wave Plus Local Orbitals Program for Calculating Crystal Properties, Vienna University of Technology, Austria, 2001.

[52] G. Kotliar, S. Y. Savrasov, K. Haule, V. S. Oudovenko, O. Parcollet, and C. A. Marianetti, Rev. Mod. Phys. 78, 865 (2006).

[53] K. Haule, Phys. Rev. B 75, 155113 (2007).

[54] P. Werner, A. Comanac, L. de'Medici, M. Troyer, and A. J. Millis, Phys. Rev. Lett. 97, 076405 (2006).
[55] K. Haule, C. H. Yee, and K. Kim, Phys. Rev. B 81, 195107 (2010).

[56] A. Kutepov, K. Haule, S. Y. Savrasov, and G. Kotliar, Phys. Rev. B 82, 045105 (2010).

[57] J. Mravlje, M. Aichhorn, T. Miyake, K. Haule, G. Kotliar, and A. Georges, Phys. Rev. Lett. 106, 096401 (2011).

[58] P. W. Selwood, Magnetochemistry (Interscience, New York, 1956).

[59] R. Kubo and H. Fukuyama, in Proceedings of the 10th International Conference on Physics of Semiconductors, Cambridge, Massachussets, 1969 (United States Atomic Energy Commission, Germantown, MD, 1970).

[60] M. L. Cohen and D. J. Chadi, in Handbook on Semiconductors, Vol. 2, edited by M. Balkanski (North-Holland, Amsterdam, 1980), p. 155 and references therein.

[61] K. Dash and G. S. Tripathi, J. Magn. Magn. Mater. 324, 602 (2012).

[62] C. S. Lue, W. J. Lai, and Y.-K. Kuo, J. Alloys Compd. 392, 72 (2005).

[63] B. Shklovskii and A. Efros, Electronic Properties of Doped Semiconductors (Springer, Berlin, 1984).

[64] See Supplemental Material at http://link.aps.org/supplemental/ 10.1103/PhysRevB.89.195102 for two figures. Figure S1 shows typical scanning electron microscope (SEM) images of $\mathrm{FeGa}_{3}$ recorded with the backscattered electron detector. The SEM study did not detect impurity phases. Figure S2 presents the results of our study on the magnetic anisotropy in $\mathrm{Fe}_{0.94} \mathrm{Mn}_{0.06} \mathrm{Ga}_{3}$. This investigation shows that the easy magnetization axis is along the [001] direction while there is only weak magnetic anisotropy within the $a b$ planes.

[65] T. Vojta, J. Phys. A: Math. Gen. 39, R143 (2006).

[66] N. Haldolaarachchige, Ph.D. thesis, Department of Physics and Astronomy, Louisiana State University, 2012.

[67] B. Kotur, V. Babizhetskyy, E. Bauer, F. Kneidinger, A. Danner, L. Leber, and H. Michor, Mater. Sci. 49, 211 (2013).

[68] Y. Takagiwa, K. Kitahara, Y. Matsubayashi, and K. Kimura, J. Appl. Phys. 111, 123707 (2012).

[69] G. Shirane, J. E. Fischer, Y. Endoh, and K. Tajima, Phys. Rev. Lett. 59, 351 (1987).

[70] J. W. Simonson, Z. P. Yin, M. Pezzoli, J. Guo, J. Liu, K. Post, A. Efimenko, N. Hollmann, Z. Hu, H.-J. Lin, C.-T. Chen, C. Marques, V. Leyva, G. Smith, J. W. Lynn, L. L. Sun, G. Kotliar, D. N. Basov, L. H. Tjeng, and M. C. Aronson, Proc. Natl. Acad. Sci. USA 109, E1815 (2012).

[71] B. C. Sales, A. F. May, M. A. McGuire, M. B. Stone, D. J. Singh, and D. Mandrus, Phys. Rev. B 86, 235136 (2012).

[72] H. G. M. Duijn, Ph.D. dissertation, Faculty of Science, University of Amsterdam, 2000.

[73] P. Bonville, F. Rullier-Albenque, D. Colson, and A. Forget, Europhys. Lett. 89, 67008 (2010).

[74] M. Neff, K. Doll, and G. Zwicknagl, J. Phys.: Condens. Matter 18, 7437 (2006).

[75] H. Watanabe, H. Yamamoto, and K. Ito, J. Phys. Soc. Jpn. 18, 995 (1963).

[76] M. P. Sarachik, D. R. He, W. Li, M. Levy, and J. S. Brooks, Phys. Rev. B 31, 1469 (1985); H. G. Schlager and H. v. Löhneysen, Europhys. Lett. 40, 661 (1997) and references therein; M. Lakner and H. v. Löhneysen, Phys. Rev. Lett. 63, 648 (1989); Y. Ootuka and N. Matsunaga, J. Phys. Soc. Jpn. 59, 1801 (1990); M. Straub, K. Kirch, and H. v. Löhneysen, 
Z. Phys. B: Condens. Matter 95, 31 (1994); S. Wagner, M. Lakner, and H. v. Löhneysen, Phys. Rev. B 55, 4219 (1997).

[77] R. N. Bhatt and P. A. Lee, J. Appl. Phys. 52, 1703 (1981); Phys. Rev. Lett. 48, 344 (1982).

[78] J. F. DiTusa, K. Friemelt, E. Bucher, G. Aeppli, and A. P. Ramirez, Phys. Rev. Lett. 78, 2831 (1997).

[79] N. Manyala, J. F. DiTusa, G. Aeppli, and A. P. Ramirez, Nature (London) 454, 976 (2008).

[80] Y. Onose, N. Takeshita, C. Terakura, H. Takagi, and Y. Tokura, Phys. Rev. B 72, 224431 (2005).
[81] R. Hu, V. F. Mitrovic, and C. Petrovic, Phys. Rev. B 79, 064510 (2009).

[82] J. Janaki, A. Mani, A. T. Satya, T. G. Kumary, S. Kalavathi, and A. Bharathi, AIP Conf. Proc. 1447, 929 (2012).

[83] A. R. Nagaraja, N. H. Perry, T. O. Mason, T. R. Paudel, Y. Tang, S. Lany, M. Grayson, and A. Zunger, J. Am. Ceram. Soc. 95, 269 (2012).

[84] S. Lany and A. Zunger, Phys. Rev. B 80, 085202 (2009).

[85] J. M. Tomczak, F. Aryasetiawan, and S. Biermann, Phys. Rev. B 78, 115103 (2008); T. Maier, M. Jarrell, T. Pruschke, and M. H. Hettler, Rev. Mod. Phys. 77, 1027 (2005). 